\title{
Decomposition of Schramm-Loewner evolution along its curve
}

\author{
Dapeng Zhan* \\ Michigan State University
}

August 26, 2018

\begin{abstract}
We show that, for $\kappa \in(0,8)$, the integral of the laws of two-sided radial $\mathrm{SLE}_{\kappa}$ curves through different interior points against a measure with $\mathrm{SLE}_{\kappa}$ Green's function density is the law of a chordal $\mathrm{SLE}_{\kappa}$ curve, biased by the path's natural length. We also show that, for $\kappa>0$, the integral of the laws of extended $\operatorname{SLE}_{\kappa}(-8)$ curves through different interior points against a measure with a closed formula density restricted in a bounded set is the law of a chordal $\mathrm{SLE}_{\kappa}$ curve, biased by the path's capacity length restricted in that set. Another result is that, for $\kappa \in(4,8)$, if one integrates the laws of two-sided chordal $\mathrm{SLE}_{\kappa}$ curves through different force points on $\mathbb{R}$ against a measure with density on $\mathbb{R}$, then one also gets a law that is absolutely continuous w.r.t. that of a chordal $\mathrm{SLE}_{\kappa}$ curve. To obtain these results, we develop a framework to study stochastic processes with random lifetime, and improve the traditional Girsanov's Theorem.
\end{abstract}

Keywords: SLE, Girsanov's Theorem, Doob-Meyer decomposition

\section{Introduction}

The Schramm-Loewner evolution (SLE), first introduced by Oded Schramm in 1999 ([26]), is a one-parameter $(\kappa \in(0, \infty))$ family of measures on non-self-crossing curves, which has received a lot of attention over the past eighteen years. It has been shown that, modulo time parametrization, several discrete random paths on grids (e.g., loop-erased random walk [16, critical percolation explorer [28, 6]) have SLE as a scaling limit.

SLE is defined using Loewner's differential equation, and is originally parameterized by capacity. For the discrete random paths which converge to SLE, in order to show the convergence, people have to first reparameterize them by capacity and then prove that the reparametrized curve converge to SLE with capacity parametrization. The convergence does not take into consideration the discrete length of the path.

In order to upgrade the convergence results, Lawler and Sheffield introduced the natural parametrization of SLE in [15], and conjectured that those discrete random paths with their

\footnotetext{
${ }^{*}$ Research partially supported by NSF grants DMS-1056840 and Sloan fellowship
} 
original length suitably rescaled, converge to the SLE with natural parametrization. Their construction used the Doob-Meyer decomposition, and they proved the existence of the natural parametrization of $\mathrm{SLE}_{\kappa}$ for $\kappa<5.021 \ldots$. This result was later improved by 20, where it was shown that the natural parametrization of $\mathrm{SLE}_{\kappa}$ exists for all $\kappa \in(0,8)$.

It was proved later in [14 that the natural parametrization agrees with the $d$-dimensional Minkowski content of the $\mathrm{SLE}_{\kappa}$ curve, where $d=1+\frac{\kappa}{8}$ is the Hausdorff dimension of the curve (cf. [4]). It was proved recently (cf. [17, 18, 19]) that the loop-erased random walk with natural length converges to $\mathrm{SLE}_{2}$ with natural parametrization. The work uses an earlier result on the convergence of the density of the loop-erased random walk to the Green's function for $\mathrm{SLE}_{2}$ (cf. [5]).

There are two major versions of SLE: chordal SLE and radial SLE. Most of the study focuses on chordal SLE, which describes a curve in a simply connected domain from one prime end (cf. 1] ) to another prime end. Two-sided radial $\mathrm{SLE}_{\kappa}$ and $\mathrm{SLE}_{\kappa}$ Green's function for $\kappa \in(0,8)$ were introduced in [15]. A two-sided radial $\mathrm{SLE}_{\kappa}$ curve has two arms: the first arm from a prime end to an interior point is a chordal $\operatorname{SLE}_{\kappa}(\kappa-8)$ process, and the second arm from the interior point to another prime end is a chordal $\mathrm{SLE}_{\kappa}$ curve conditioned on the first arm. It can be understood as a chordal $\mathrm{SLE}_{\kappa}$ curve conditioned to pass through a fixed interior point. While that event has probability zero, some limiting procedure was used to make this idea rigorous. $\mathrm{SLE}_{\kappa}$ Green's function was defined by a closed formula, and turned out to be the density of the $\mathrm{SLE}_{\kappa}$ curve with natural parametrization. These two objects have discrete analogues. The two-sided radial $\mathrm{SLE}_{\kappa}$ curve corresponds to the discrete random path conditioned to pass through a fixed vertex, and the $\mathrm{SLE}_{\kappa}$ Green's function corresponds to the density of the path.

Field recently proved in $[8$ that, for $\kappa \in(0,4]$, if one integrates the laws of two-sided radial $\mathrm{SLE}_{\kappa}$ in a bounded analytic domain $D$ passing through different interior points (with the two ends fixed) against the measure with density (w.r.t. the Legesgue measure on $\mathbb{C}$ ) being the $\mathrm{SLE}_{\kappa}$ Green's function in $D$, then one gets the law of a chordal $\mathrm{SLE}_{\kappa}$ curve biased by the curve's length in the natural parametrization. This is analogous to a simple fact of discrete random paths: if one integrates the laws of the path conditioned to pass through different fixed vertices against the probability that the path passes through each fixed vertex, one should get a measure on paths, which is absolutely continuous w.r.t. the law of the original discrete random path, and the Radon-Nikodym derivative is the total number of vertices on the path, which is due to the repetition of counting.

This paper is motivated by Field's work. We extend his result from $\kappa \in(0,4]$ to $\kappa \in(0,8)$ (see Corollary 4.3) using a new approach. We do not need to assume that the domain is bounded or analytic. The main tools used here are from Probability Theory.

Our approach is different from Field's in that we develop a theory of stochastic processes with random lifetime, which is purely probability. The computation here is simpler because the major work: the Doob-Meyer decomposition for the definition of the natural parametrization was done earlier in [15, 20. On the other hand, Field's proof used some SLE technique such as an escape estimate of SLE derived in his joint work 9] with Lawler. That results in the technical assumption in his paper that $\kappa \leq 4$ and that the domain is bounded with analytic boundary. Another difference is that we study the measures on the space of curve-point pairs instead of just on curves. This makes the main theorem more convenient to be applied later in [33] to construct SLE loop measures.

After the main theorem, we find other applications of the new technique. We define the extended chordal $\operatorname{SLE}_{\kappa}(\rho)$ curve in the upper half plane $\mathbb{H}$ for $\rho \leq \frac{\kappa}{2}-4$, which is composed 
of two arms: the first arm growing from 0 to $z_{0} \in \mathbb{H}$ is a chordal $\operatorname{SLE}_{\kappa}(\rho)$ curve, and the second arm growing from $z_{0}$ to $\infty$ is a chordal $\mathrm{SLE}_{\kappa}$ curve conditioned on the first arm. In particular, a two-sided radial $\mathrm{SLE}_{\kappa}$ curve is an extended chordal $\mathrm{SLE}_{\kappa}(\kappa-8)$ curve. We prove that, for any $\kappa>0$, there is a positive function $G^{\kappa,-8}(z)$ with closed formula such that for any measurable $U \subset \mathbb{H}$ on which $G^{\kappa,-8}$ is integrable, if one integrates the laws of extended chordal $\operatorname{SLE}_{\kappa}(-8)$ curve through different $z$ against a measure with density $\mathbf{1}_{U} G^{\kappa,-8}(z)$, then one gets the law of a chordal $\mathrm{SLE}_{\kappa}$ curve biased by $C_{\kappa, 1}$ times the total time that the curve spends in $U$ in the capacity parametrization (see Corollary 5.1), where $C_{\kappa, 1}$ is a positive constant depending only on $\kappa$.

The above two main results of this paper immediately imply that, if we sample a point on a chordal $\mathrm{SLE}_{\kappa}$ curve in $\mathbb{H}$ according to a law, which is absolutely continuous w.r.t. the natural parametrization (resp. capacity parametrization), and stop the curve at that point, then we get a random curve, whose law is absolutely continuous w.r.t. that of a chordal $\operatorname{SLE}_{\kappa}(\kappa-8)\left(\right.$ resp. $\left.\operatorname{SLE}_{\kappa}(-8)\right)$ curve in $\mathbb{H}$.

The connection between $\operatorname{SLE}_{\kappa}$ near its tip at a fixed capacity time and $\operatorname{SLE}_{\kappa}(-8)$ was derived earlier in [7, Proposition 3.10]. The tip behavior of SLE was also studied in [32] for $\kappa \in(0,4)$. What new here is that we derive the Green's function for the SLE in capacity parametrization up to a multiplicative constant.

Another application of the new technique is to study the intersection of SLE curve with the boundary. Using a Doob-Meyer decomposition, Alberts and Sheffield constructed in 2] a measure supported by the intersection of an $\operatorname{SLE}_{\kappa}$ curve $\gamma$ in $\mathbb{H}$ for $\kappa \in(4,8)$ with $\mathbb{R}$, and conjectured that the measure agrees with the $\left(2-\frac{8}{\kappa}\right)$-dimensional Minkowski content of $\gamma \cap \mathbb{R}$. Their work used two-sided chordal $\mathrm{SLE}_{\kappa}$, which can be understood as a chordal $\mathrm{SLE}_{\kappa}$ curve conditioned to pass through a fixed point on the boundary. Using their result, we prove in this paper that, if one integrates the laws of two-sided chordal $\mathrm{SLE}_{\kappa}$ curve in $\mathbb{H}$ from 0 to $\infty$ through different $x \in \mathbb{R}$ against a measure with a suitable density function (known as boundary Green's function for SLE) against the Lebesgue measure on a bounded interval $I \subset \mathbb{R}$, then one gets a measure on curves, which is absolutely continuous w.r.t. the law of a chordal $\mathrm{SLE}_{\kappa}$ curve, and the Radon-Nikodym derivative is the measure constructed in 2] of the intersection of the curve with $I$.

The new technique has applications beyond the SLE area. For example, we use it to decompose a planar Brownian motion restricted in a simply connected domain.

In the author's recent preprint 33, the decomposition of chordal SLE into two-sided radial SLE is used to construct SLE loop measures.

The paper is organized as follows. In Section 2 in order to study the driving functions of $\operatorname{SLE}_{\kappa}(\rho)$ curves, we develop a framework on stochastic processes with random lifetime. We introduce the "local absolute continuity" between these processes, and extend the traditional Girsanov's theorem. In Section 3, we review the definitions and basic properties of $\mathrm{SLE}_{\kappa}$ and its variants, $\operatorname{SLE}_{\kappa}(\rho)$ processes. In Section 4 , we prove our main result about natural parametrization and two-sided radial SLE curves. In Section [5 we prove the result about capacity parametrization and extended chordal $\operatorname{SLE}_{\kappa}(-8)$ curves. In Section 6 , we show the application on boundary measure and two-sided chordal SLE curves. In Section 7, we use the technique to decompose planar Brownian motions. In the appendix, we prove the transience property of chordal $\operatorname{SLE}_{\kappa}(\rho)$ curves. 


\section{Acknowledgement}

The author acknowledges the support from the National Science Foundation under the grant DMS-1056840 and the support from the Alfred P. Sloan Foundation.

\section{Stochastic Processes with Random Lifetime}

For $0<T \leq \infty$, let $C([0, T))$ denote the space of real valued continuous functions on $[0, T)$. Let

$$
\Sigma=\bigcup_{0<T \leq \infty} C([0, T)) .
$$

For each $f \in \Sigma$, let $T_{f}$ be such that $\left[0, T_{f}\right)$ is the domain of $f$.

We define two basic operations on $\Sigma$ : killing and continuing. For $0<\tau \leq \infty$, we define the killing map $\mathcal{K}_{\tau}: \Sigma \rightarrow \Sigma$ such that if $g=\mathcal{K}_{\tau}(f)$, then $T_{g}=\tau \wedge T_{f}$ and $g=\left.f\right|_{\left[0, T_{g}\right)}$. Let $\Sigma^{\oplus}=\left\{f \in \Sigma: T_{f}<\infty, f\left(T_{f}^{-}\right):=\lim _{t \rightarrow T_{f}^{-}} f(t) \in \mathbb{R}\right\}$ and $\Sigma_{\oplus}=\{f \in \Sigma: f(0)=0\}$. For example, if $T_{f}>\tau>0$, then $\mathcal{K}_{\tau}(f) \in \Sigma^{\oplus}$. For $f \in \Sigma^{\oplus}$ and $g \in \Sigma_{\oplus}$, we may continue $f$ using $g$ and get the function $f \oplus g \in \Sigma$, which is defined by $T_{f \oplus g}=T_{f}+T_{g}$ and

$$
f \oplus g(t)= \begin{cases}f(t), & 0 \leq t<T_{f} \\ f\left(T_{f}^{-}\right)+g\left(t-T_{f}\right), & T_{f} \leq t<T_{f}+T_{g}\end{cases}
$$

Sometimes we want to record the time that the two functions are joined together. For this purpose, we define $f \widehat{\oplus} g=\left(f \oplus g, T_{f}\right)$. Then we can use $f \widehat{\oplus} g$ to recover $f$ and $g$.

For $0 \leq t<\infty$, let $\mathcal{F}_{t}^{0}$ be the $\sigma$-algebra generated by

$$
\left\{f \in \Sigma: s<T_{f}, f(s) \in U\right\}, \quad 0 \leq s \leq t, U \in \mathcal{B}(\mathbb{R}) .
$$

Then $\left(\mathcal{F}_{t}^{0}\right)$ is a filtration on $\Sigma$. Let $\mathcal{F}^{0}=\sigma\left(\bigcup_{0<t<\infty} \mathcal{F}_{t}^{0}\right)$. We will mainly work on the measurable space $\left(\Omega, \mathcal{F}^{0}\right)$ or its completion w.r.t. a certain measure. Every probability measure on $\left(\Sigma, \mathcal{F}^{0}\right)$ is the law of a continuous stochastic process with random lifetime. For any measure $\mu$ on $\left(\Sigma, \mathcal{F}^{0}\right)$, we use $\mathcal{F}^{\mu}$ and $\mathcal{F}_{t}^{\mu}$ to denote the $\mu$-completion of $\mathcal{F}^{0}$ and $\mathcal{F}_{t}^{0}$, respectively.

The continuing maps $(f, g) \mapsto f \oplus g$ and $(f, g) \mapsto f \widehat{\oplus} g$ are measurable. If $\mu$ and $\nu$ are $\sigma$-finite measures supported by $\Sigma^{\oplus}$ and $\Sigma_{\oplus}$, respectively, we use $\mu \oplus \nu$ and $\mu \widehat{\oplus} \nu$ to denote the pushforward measures of the product measure $\mu \otimes \nu$ under the maps $(f, g) \mapsto f \oplus g$ and $(f, g) \mapsto f \widehat{\oplus} g$, respectively.

Let's recall an important notion in Probability: kernel. Suppose $(U, \mathcal{U})$ and $(V, \mathcal{V})$ are two measurable spaces. A kernel from $(U, \mathcal{U})$ to $(V, \mathcal{V})$ is a map $\nu: U \times \mathcal{V} \rightarrow[0, \infty]$ such that (i) for every $u \in U, \nu(u, \cdot)$ is a measure on $(V, \mathcal{V})$, and (ii) for every $F \in \mathcal{V}, \nu(\cdot, F)$ is $\mathcal{U}$-measurable. Let $\mu$ be a $\sigma$-finite measure on $(U, \mathcal{U})$. Let $\mathcal{U}^{\mu}$ be the $\mu$-completion of $\mathcal{U}$. A $\mu$-kernel from $(U, \mathcal{U})$ to $(V, \mathcal{V})$ is a kernel from $\left(U^{\mu}, \mathcal{U}^{\mu} \cap U^{\mu}\right)$ to $(V, \mathcal{V})$, where $U^{\mu} \subset U$ is such that $U \backslash U^{\mu}$ is a $\mu$-null. The $\mu$-kernel is said to be finite if for $\mu$-a.s. every $u \in U$, $\nu(u, V)<\infty$; and is said to be $\sigma$-finite if there is a sequence $F_{n} \in \mathcal{V}, n \in \mathbb{N}$, with $V=\bigcup F_{n}$ such that for any $n \in \mathbb{N}$, and $\mu$-a.s. every $u \in U, \nu\left(u, F_{n}\right)<\infty$. If $\nu$ is a $\sigma$-finite $\mu$-kernel from $(U, \mathcal{U})$ to $(V, \mathcal{V})$, then we may define a measure $\mu \otimes \nu$ on $\mathcal{U} \times \mathcal{V}$ such that

$$
\mu \otimes \nu(E \times F)=\int_{E} \nu(u, F) d \mu(u), \quad E \in \mathcal{U}, \quad \mathcal{F} \in \mathcal{V} .
$$


This new measure is first defined on the semi-ring $\{E \times F: E \in \mathcal{U}, \mathcal{F} \in \mathcal{V}\}$, and then extended to a measure on $\mathcal{U} \times \mathcal{V}$. Carathéodory's extension theorem guarantees the existence of the extension. The $\sigma$-finiteness of $\mu$ and $\nu$ ensures that the extension is unique, and $\mu \otimes \nu$ is also $\sigma$-finite. We use $\mu \cdot \nu$ to denote the marginal of $\mu \otimes \nu$ on $(V, \mathcal{V})$, i.e., $\mu \cdot \nu(F)=$ $\int_{U} \nu(u, F) d \mu(u), F \in \mathcal{V}$. If $\nu$ is a $\sigma$-finite measure on $(V, \mathcal{V})$, and $\mu$ is a $\sigma$-finite $\nu$-kernel from $(V, \mathcal{V})$ to $(U, \mathcal{U})$, then we use $\mu \overleftarrow{\otimes} \nu$ to denote the pushforward measure on $\mathcal{U} \times \mathcal{V}$ of $\nu \otimes \mu$ under the map $(v, u) \mapsto(u, v)$.

The killing map $\mathcal{K}:(f, r) \mapsto \mathcal{K}_{r}(f)$ is also measurable. If $\nu$ is a $\sigma$-finite $\mu$-kernel from $\Sigma$ to $(0, \infty)$, we use $\mathcal{K}_{\nu}(\mu)$ to denote the pushforward measure of $\mu \otimes \nu$ under $\mathcal{K}$.

Let $\Sigma_{t}=\left\{f \in \Sigma: T_{f}>t\right\}, t \geq 0$. Then $\left(\Sigma_{t}\right)$ is a decreasing family of subspaces of $\Sigma$ with $\Sigma_{0}=\Sigma$ and $\Sigma_{\infty}:=\cap_{t=0}^{\infty} \Sigma_{t}=C([0, \infty))$. We will be interested in the restriction of $\mathcal{F}_{t}^{0}$ to $\Sigma_{t}$, i.e., $\mathcal{F}_{t}^{0} \cap \Sigma_{t}$. Note that $\bigcup_{0 \leq t<\infty} \mathcal{F}_{t}^{0} \cap \Sigma_{t}$ is a $\pi$-system, which generates the $\sigma$-algebra $\mathcal{F}^{0}$, and $\Sigma=\Sigma_{0} \in \mathcal{F}_{0}^{0} \cap \Sigma_{0}$. This enables us to apply Dynkin's $\pi-\lambda$ theorem. For example, if two finite measures on $\left(\Sigma, \mathcal{F}^{0}\right)$ agree on each $\mathcal{F}_{t}^{0} \cap \Sigma_{t}$, then they are equal.

Let $\mu$ and $\nu$ be two measures on $\left(\Sigma, \mathcal{F}^{0}\right)$, which are $\sigma$-finite on $\mathcal{F}_{0}^{0}$ (and so are $\sigma$-finite on each $\mathcal{F}_{t}^{0}$ ). We say that $\nu$ is locally absolutely continuous w.r.t. $\mu$, and write $\nu \triangleleft \mu$, if for any $0 \leq t<\infty,\left.\nu\right|_{\mathcal{F}_{t}^{0} \cap \Sigma_{t}}$ is absolutely continuous w.r.t. $\left.\mu\right|_{\mathcal{F}_{t}^{0} \cap \Sigma_{t}}$. This is certainly the case if $\nu \ll \mu$, i.e., $\nu$ is (globally) absolutely continuous w.r.t. $\mu$. The process $M_{t}:=\frac{\left.d \nu\right|_{\mathcal{F}_{t}^{0} \cap \Sigma_{t}}}{\left.d \mu\right|_{\mathcal{F}_{t}^{0} \cap \Sigma_{t}}}$, $0 \leq t<\infty$, is called the local Radon-Nikodym derivative of $\nu$ w.r.t. $\mu$. By Dynkin's $\pi-\lambda$ theorem, we see that $\nu$ is determined by $\mu$ and $\left(M_{t}\right)$. Thus, we say that $\nu$ can be obtained by locally weighting $\mu$ by $\left(M_{t}\right)$.

Proposition 2.1. Let $\mu$ be a measure on $\left(\Sigma, \mathcal{F}^{0}\right)$, which is $\sigma$-finite on $\mathcal{F}_{0}^{0}$. Let $(\Upsilon, \mathcal{G})$ be a measurable space. Let $\nu: \Upsilon \times \mathcal{F}^{0} \rightarrow[0, \infty]$ be such that for every $v \in \Upsilon, \nu(v, \cdot)$ is a finite measure on $\mathcal{F}^{0}$ that is locally absolutely continuous w.r.t. $\mu$. Moreover, suppose that the local Radon-Nikodym derivatives are equal to $\left(M_{t}(v, \cdot)\right)$, where $M_{t}:(\Upsilon, \mathcal{G}) \times\left(\Sigma, \mathcal{F}_{t}\right) \rightarrow[0, \infty)$ is measurable for every $t \geq 0$. Then $\nu$ is a kernel from $(\Upsilon, \mathcal{G})$ to $\left(\Sigma, \mathcal{F}^{0}\right)$. Moreover, if $\xi$ is a $\sigma$-finite measure on $(\Upsilon, \mathcal{G})$ such that $\mu$-a.s., $\int_{\Upsilon} M_{t}(v, \cdot) d \xi(v)<\infty$ for all $t \geq 0$, then $\xi \cdot \nu \triangleleft \mu$, and the local Radon-Nikodym derivatives are $\int_{\Upsilon} M_{t}(v, \cdot) d \xi(v), 0 \leq t<\infty$.

Proof. By Dynkin's $\pi-\lambda$ theorem, to prove that $v \mapsto \nu(v, \cdot)$ is measurable, it suffices to show that, for any $t \in[0, \infty)$ and any $A \in \mathcal{F}_{t}^{0} \cap \Sigma_{t}, v \mapsto \nu(v, A)$ is measurable, which easily follows from Tonelli's theorem because $\nu(v, A)=\int_{A} M_{t}(v, f) d \mu(f)$. To prove that $\xi \cdot \nu \triangleleft \mu$ and find the local Radon-Nikodym derivatives, we apply Tonelli's theorem again and get

$$
\xi \cdot \nu(A)=\int_{\Upsilon} \nu(v, A) d \xi(v)=\int_{\Upsilon} \int_{A} M_{t}(v, f) d \mu(f) d \xi(v)=\int_{A} \int_{\Upsilon} M_{t}(v, f) d \xi(v) d \mu(f)
$$

for $A \in \mathcal{F}_{t}^{0} \cap \Sigma_{t}$. Then we conclude that $\xi \cdot \nu \triangleleft \mu$, and conclude that the local RadonNikodym derivatives are $\int_{\Upsilon} M_{t}(v, \cdot) d \xi(v), 0 \leq t<\infty$.

Proposition 2.2. Let $\mu$ be a probability measure on $\left(\Sigma, \mathcal{F}^{0}\right)$. Let $\xi$ be a $\mu$-kernel from $\left(\Sigma, \mathcal{F}^{0}\right)$ to $(0, \infty)$ that satisfies $\mathbb{E}_{\mu}[|\xi|]<\infty$. Then $\mathcal{K}_{\xi}(\mu) \triangleleft \mu$, and the local Radon-Nikodym derivatives are $\mathbb{E}_{\mu}\left[\xi((t, \infty)) \mid \mathcal{F}_{t}^{0}\right], 0 \leq t<\infty$.

Proof. Fix $t \in[0, \infty)$, and $E \in \mathcal{F}_{t}^{0} \cap \Sigma_{t}$. It is easy to see that $\mathcal{K}_{r}(f) \in E$ iff $f \in E$ and $r>t$, which implies that

$$
\mathcal{K}_{\xi}(\mu)(E)=\mu \otimes \xi(E \times(t, \infty))=\int_{E} \xi(f,(t, \infty)) d \mu(f)=\int_{E} \mathbb{E}_{\mu}\left[\xi((t, \infty)) \mid \mathcal{F}_{t}^{0}\right] d \mu .
$$


Then we get the conclusion.

Remark. Proposition 2.2 will be mainly applied to the case that $\xi=d \theta$, where $\left(\theta_{t}\right)$ is an $\left(\mathcal{F}_{t}^{\mu}\right)$-adapted right-continuous increasing process with $\theta_{0}=\theta_{0^{-}}=0$ and $\mathbb{E}_{\mu}\left[\theta_{\infty}\right]<\infty$. Applying the proposition, we find that $\mathcal{K}_{d \theta}(\mu) \triangleleft \mu$, and

$$
\mu-\text { a.s., } \quad \frac{\left.d \mathcal{K}_{d \theta}(\mu)\right|_{\mathcal{F}_{t}^{0} \cap \Sigma_{t}}}{\left.d \mu\right|_{\mathcal{F}_{t}^{0} \cap \Sigma_{t}}}=\mathbb{E}_{\mu}\left[\theta_{\infty} \mid \mathcal{F}_{t}^{\mu}\right]-\theta_{t} .
$$

Fix $\kappa>0$. Let $\mathbb{P}_{\kappa}$ be the law of $\sqrt{\kappa}$ times a standard Brownian motion. This means that $\frac{1}{\sqrt{\kappa}}$ times the coordinate process on $\Sigma$ under $\mathbb{P}_{\kappa}$ is a standard Brownian motion. For this reason, we use $\left(B_{t}\right)$ to denote the above standard Brownian motion on $\Sigma$, i.e., $\frac{1}{\sqrt{\kappa}}$ times the coordinate process. We observe that $\mathbb{P}_{\kappa}$ is supported by $\Sigma_{\infty} \cap \Sigma_{\oplus}$. Let $\mathcal{F}_{t}^{B}$ and $\mathcal{F}^{B}$ be the $\mathbb{P}_{\kappa}$-completion of $\mathcal{F}_{t}^{0}$ and $\mathcal{F}^{0}$, respectively; and $\mathbb{E}_{\kappa}$ denote the expectation w.r.t. $\mathbb{P}_{\kappa}$.

We now use Girsanov's theorem to derive local Radon-Nikodym derivatives. Recall that when we used Girsanov's theorem to weight a probability measure by a positive local martingale, we had to stop the process at some stopping time to get a bounded martingale. The following proposition says that we do not need to do the stopping, and the local martingale valued at different times are just the local Radon-Nikodym derivatives.

Proposition 2.3. Suppose that $\left(X_{t}\right)_{0 \leq t<T_{0}}$ satisfies $X_{0}=0$ and the $\left(\mathcal{F}_{t}^{B}\right)$-adapted SDE:

$$
d X_{t}=\sqrt{\kappa} d B_{t}+\sigma_{t} d t, \quad 0 \leq t<T_{0},
$$

where $T_{0}$ is a positive $\left(\mathcal{F}_{t}^{B}\right)$-stopping time and $\left(\sigma_{t}\right)_{0 \leq t<T_{0}}$ is a real valued $\left(\mathcal{F}_{t}^{B}\right)$-adapted continuous process. Let $\mathbb{P}^{\kappa, \sigma}$ denote the law of $X$. Then $\mathbb{P}^{\kappa, \sigma} \triangleleft \mathbb{P}_{\kappa}$. Moreover, if $M_{t}$, $0 \leq t<T_{0}$, is an $\left(\mathcal{F}_{t}^{B}\right)$-adapted continuous local martingale that satisfies $M_{0}=1$ and the $S D E$ :

$$
d M_{t}=M_{t} \frac{\sigma_{t}}{\sqrt{\kappa}} d B_{t}, \quad 0 \leq t<T_{0} .
$$

then

$$
\frac{\left.d \mathbb{P}^{\kappa, \sigma}\right|_{\mathcal{F}_{t}^{0} \cap \Sigma_{t}}}{\left.d \mathbb{P}_{\kappa}\right|_{\mathcal{F}_{t}^{0} \cap \Sigma_{t}}}=\mathbf{1}_{T_{0}>t} M_{t}, \quad 0 \leq t<\infty
$$

Proof. Let $M_{t}=\exp \left(\int_{0}^{t} \frac{\sigma_{s}}{\sqrt{\kappa}} d B_{s}-\int_{0}^{t} \frac{\sigma_{s}^{2}}{2 \kappa} d s\right), 0 \leq t<T_{0}$. From Itô's formula (cf. [23]), we see that $\left(M_{t}\right)$ satisfies $M_{0}=1$ and (2.2). Thus, it suffices to prove (2.3).

Fix $N \in \mathbb{N}$ and let $\tau_{N}=\inf \left\{0 \leq t<T_{0}:\left|M_{t}\right| \geq N\right\}$. Here we set $\inf \emptyset=T_{0}$. Then $\tau_{N}$ is a stopping time with $\tau_{N} \leq T_{0}$, and $M_{t}, 0 \leq t<\tau_{N}$, is uniformly bounded by $N$. If $\tau_{N}=T_{0}$, then $\mathbb{P}_{\kappa}$-a.s. $\lim _{t \rightarrow T_{0}^{-}} M_{t}$ exists. Let

$$
M_{t}^{\tau_{N}}:= \begin{cases}M_{t \wedge \tau_{N}}, & t \wedge \tau_{N}<T_{0} \\ \liminf _{s \rightarrow T_{0}^{-}} M_{s}, & t \geq \tau_{N}=T_{0}\end{cases}
$$

Then $\left(M_{t}^{\tau_{N}}, 0 \leq t<\infty\right)$ is a uniformly bounded $\left(\mathbb{P}_{\kappa}\right.$-a.s. $)$ continuous local martingale, and satisfies the SDE:

$$
d M_{t}^{\tau_{N}}=M_{t}^{\tau_{N}} \mathbf{1}_{t<\tau_{N}} \frac{\sigma_{t}}{\sqrt{\kappa}} d B_{t}, \quad 0 \leq t<\infty .
$$


Let $M_{\infty}^{\tau_{N}}=\liminf _{t \rightarrow \infty} M_{t}^{\tau_{N}}$. Then for any $0 \leq t<\infty$, a.s. $\mathbb{E}_{\kappa}\left[M_{\infty}^{\tau_{N}} \mid \mathcal{F}_{t}^{B}\right]=M_{t}^{\tau_{N}}$. In particular, since $M_{0}^{\tau_{N}}=1$, we have $\mathbb{E}_{\kappa}\left[M_{\infty}^{\tau_{N}}\right]=1$. Define $\mathbb{P}_{N}^{\kappa, \sigma}$ such that $\frac{d \mathbb{P}_{N}^{\kappa, \sigma}}{d \mathbb{P}_{\kappa}}=M_{\infty}^{\tau_{N}}$. Then $\mathbb{P}_{\kappa}^{N}$ is also a probability measure on $\left(\Sigma, \mathcal{F}^{0}\right)$. Let

$$
B_{t}^{N}=B_{t}-\int_{0}^{\tau_{N} \wedge t} \frac{\sigma_{s}}{\sqrt{\kappa}} d s, \quad 0 \leq t<\infty .
$$

From Girsanov's theorem (cf. [23]) and (2.4), we know that the law of $B^{N}$ under $\mathbb{P}_{N}^{\kappa, \sigma}$ is also that of a standard Brownian motion. From

$$
\sqrt{\kappa} B_{t}=\sqrt{\kappa} B_{t}^{N}+\int_{0}^{t} \sigma_{s} d s, \quad 0 \leq t<\tau_{N} .
$$

we see that the law of $\left(\sqrt{\kappa} B_{t}, 0 \leq t<\tau_{N}\right)$ under $\mathbb{P}_{N}^{\kappa, \sigma}$ is the same as the law of $\left(X_{t}, 0 \leq t<\right.$ $\left.\tau_{N}\right)$ under $\mathbb{P}_{\kappa}$.

Fix $t \in[0, \infty)$ and $E \in \mathcal{F}_{t}^{0} \cap \Sigma_{t}$. Since $T_{0}$ is the lifetime of $X$, and $T_{0}=\sup _{N \in \mathbb{N}} \tau_{N}$, we have

$$
X^{-1}(E) \subset X^{-1}\left(\Sigma_{t}\right) \subset\left\{T_{0}>t\right\}=\bigcup_{N \in \mathbb{N}}\left\{\tau_{N}>t\right\} .
$$

Since the law of $\left(\sqrt{\kappa} B_{t}, 0 \leq t<\tau_{N}\right)$ under $\mathbb{P}_{N}^{\kappa, \sigma}$ is the same as the law of $\left(X_{t}, 0 \leq t<\tau_{N}\right)$ under $\mathbb{P}_{\kappa}$, and $\left(\sqrt{\kappa} B_{t}\right)$ is the coordinate process, we get

$$
\begin{gathered}
\mathbb{P}_{\kappa}\left[X^{-1}(E) \cap\left\{\tau_{N}>t\right\}\right]=\mathbb{P}_{N}^{\kappa, \sigma}\left[E \cap\left\{\tau_{N}>t\right\}\right]=\mathbb{E}_{\kappa}\left[\mathbf{1}_{E \cap\left\{\tau_{N}>t\right\}} M_{\infty}^{\tau_{N}}\right] \\
=\mathbb{E}_{\kappa}\left[\mathbf{1}_{E \cap\left\{\tau_{N}>t\right\}} M_{t}^{\tau_{N}}\right]=\mathbb{E}_{\kappa}\left[\mathbf{1}_{E} \mathbf{1}_{\left\{\tau_{N}>t\right\}} M_{t}\right],
\end{gathered}
$$

where the third equality follows from the optional stopping theorem, and the last equality holds because $M_{t}^{\tau_{N}}=M_{t}$ on $\left\{\tau_{N}>t\right\}$. This together with (2.5) implies that

$$
\mathbb{P}^{\kappa, \sigma}(E)=\mathbb{P}_{\kappa}\left[X^{-1}(E)\right]=\lim _{N \rightarrow \infty} \mathbb{P}_{\kappa}\left[X^{-1}(E) \cap\left\{\tau_{N}>t\right\}\right]=\mathbb{E}_{\kappa}\left[\mathbf{1}_{E} \mathbf{1}_{\left\{T_{0}>t\right\}} M_{t}\right] .
$$

So we get (2.3) and finish the proof.

At the end of this section, we state and prove the following proposition, which extends the strong Markov property of Brownian motions.

Proposition 2.4. Let $\left(\theta_{t}\right)_{0 \leq t<\infty}$ be a right-continuous increasing $\left(\mathcal{F}_{t}^{B}\right)$-adapted process that satisfies $\theta_{0}=\theta_{0^{+}}=0$ and $\mathbb{E}_{\kappa}\left[\theta_{\infty}\right]<\infty$. Then

$$
\mathcal{K}_{d \theta}\left(\mathbb{P}_{\kappa}\right) \widehat{\oplus} \mathbb{P}_{\kappa}=\mathbb{P}_{\kappa} \otimes d \theta .
$$

Thus, $\mathcal{K}_{d \theta}\left(\mathbb{P}_{\kappa}\right) \oplus \mathbb{P}_{\kappa} \ll \mathbb{P}_{\kappa}$, and $\theta_{\infty}$ is the Radon-Nikodym derivative.

Remark. If $\theta_{t}=\mathbf{1}_{\tau \leq t}$, where $\tau$ is a positive finite $\left(\mathcal{F}_{t}^{B}\right)$-stopping time, then the proposition reduces to the strong Markov property of $\left(\sqrt{\kappa} B_{t}\right)$, i.e., $\mathcal{K}_{\delta_{\tau}}\left(\mathbb{P}_{\kappa}\right) \oplus \mathbb{P}_{\kappa}=\mathbb{P}_{\kappa}$.

Proof. First, assume that there is $t_{0} \in(0, \infty)$ and $E \in \mathcal{F}_{t_{0}}^{B}$ such that $\theta_{t}(f)=\mathbf{1}_{E}(f)$. $\mathbf{1}_{\left[t_{0}, \infty\right)}(t)$. Fix $t_{0}^{\prime} \in\left(0, t_{0}\right), A \in \mathcal{F}_{t_{0}^{\prime}}^{B}$ and $B \in \mathcal{F}^{B}$. For every $r \in[0, \infty)$, define $\mathcal{S}_{r}: \Sigma_{r} \rightarrow \Sigma_{\oplus}$ such that if $g=\mathcal{S}_{r}(f)$, then $T_{g}=T_{f}-r$, and $g(t)=f(r+t)-f(r), 0 \leq t<T_{g}$. Let 
$A \oplus_{t_{0}} B=\left\{f \in A \cap \Sigma_{t_{0}}: \mathcal{S}_{t_{0}}(f) \in B\right\}$. Since $\mathcal{S}_{t_{0}}\left(\mathcal{K}_{t_{0}}(f) \oplus g\right)=g$, and $\mathcal{K}_{t_{0}}(f) \in A$ iff $f \in A$ and $r>t_{0}^{\prime}$, we get

$\mathcal{K}_{d \theta}\left(\mathbb{P}_{\kappa}\right) \oplus \mathbb{P}_{\kappa}\left(A \oplus_{t_{0}} B\right)=\mathcal{K}_{d \theta}\left(\mathbb{P}_{\kappa}\right)(A) \mathbb{P}_{\kappa}(B)=\mathbb{P}_{\kappa} \otimes d \theta\left(A \times\left(t_{0}^{\prime}, \infty\right)\right) \mathbb{P}_{\kappa}(B)=\mathbb{P}_{\kappa}(A \cap E) \mathbb{P}_{\kappa}(B)$.

From the Markov property of $\left(\sqrt{\kappa} B_{t}\right)$, we get

$$
\int_{A \oplus t_{0} B} \theta_{\infty} d \mathbb{P}_{\kappa}=\int_{A \oplus_{0} B} \mathbf{1}_{E} d \mathbb{P}_{\kappa}=\mathbb{P}_{\kappa}\left((A \cap E) \oplus_{t_{0}} B\right)=\mathbb{P}_{\kappa}(A \cap E) \cdot \mathbb{P}_{\kappa}(B) .
$$

Define $\mathbb{P}_{\kappa}^{\theta}$ such that $d \mathbb{P}_{\kappa}^{\theta} / d \mathbb{P}_{\kappa}=\theta_{\infty}$. From the above two displayed formulas, we see that $\mathcal{K}_{d \theta}\left(\mathbb{P}_{\kappa}\right) \oplus \mathbb{P}_{\kappa}$ agrees with $\mathbb{P}_{\kappa}^{\theta}$ on the sets $A \oplus_{t_{0}} B$, where $A \in \mathcal{F}_{t_{0}^{\prime}}^{B}, t_{0}^{\prime} \in\left(0, t_{0}\right)$, and $B \in \mathcal{F}^{B}$. Since these sets form a $\pi$-system, Dynkin's $\pi-\lambda$ theorem implies that the two measures agree on the $\sigma$-algebra generated by these sets, which agrees with $\mathcal{F}^{B}$ restricted to $\Sigma_{t_{0}}$. Since both measures are supported by $\Sigma_{\infty} \subset \Sigma_{t_{0}}$, we get $\mathcal{K}_{d \theta}\left(\mathbb{P}_{\kappa}\right) \oplus \mathbb{P}_{\kappa}=\mathbb{P}_{\kappa}^{\theta}$. Since these two measures are the projections of $\mathcal{K}_{d \theta}\left(\mathbb{P}_{\kappa}\right) \widehat{\oplus} \mathbb{P}_{\kappa}$ and $\mathbb{P}_{\kappa} \otimes d \theta$, respectively, to $\Sigma$, and the projections of them to $(0, \infty)$ are both concentrated at $t_{0}$, we get (2.6) in this special case.

Second, assume that $\left(\theta_{t}\right)$ has the form of $\sum_{n=1}^{\infty} C_{n} \theta_{t}^{(n)}$, where each $C_{n}$ is a nonnegative real number and each $\theta_{t}^{(n)}$ satisfies the condition in the previous paragraph. In this case, we get (2.6) using the result in the above paragraph and the fact that both sides of (2.6) satisfy the countable linearity in $\left(\theta_{t}\right)$.

Finally, we consider the general case. Since $\mathbb{E}_{\kappa}\left[\theta_{\infty}\right]<\infty$, from the linearity of both sides of (2.6) in $d \theta$, we may assume that $\mathbb{E}_{\kappa}\left[\theta_{\infty}\right]=1$. In this case both sides of (2.6) are probability measures. For $n \in \mathbb{N}$, define $U^{(n)}$ and $L^{(n)}$ from $[0, \infty)$ to $[0, \infty)$ such that

$$
U^{(n)}(t)=\frac{\left\lfloor 2^{n} \cdot t\right\rfloor+1}{2^{n}}, \quad L^{(n)}(t)=0 \vee \frac{\left\lceil 2^{n} \cdot t\right\rceil-1}{2^{n}} .
$$

Then $U^{(n)}(t) \downarrow t$ and $L^{(n)}(t) \uparrow t$ for any $t \in[0, \infty)$. Moreover, we have

$$
L^{(n)}(t) \leq s \quad \text { if and only if } t \leq U^{(n)}(s), \quad \forall t, s \in[0, \infty) .
$$

This equivalence holds because for any $t, s \geq 0$, both sides are equivalent to that there is an integer $n$ such that $2^{n} t \leq n \leq 2^{n} s+1$. Define $\left(\theta_{t}^{(n)}\right)$ such that $\theta_{t}^{(n)}=\theta_{U^{(n)}(t)}$. Then $\left(\theta_{t}^{(n)}\right)$ has the form of that in the above paragraph since it takes values only at $k / 2^{n}, k \in \mathbb{Z}$. Thus, $\mathcal{K}_{d \theta^{(n)}}\left(\mathbb{P}_{\kappa}\right) \widehat{\oplus} \mathbb{P}_{\kappa}=\mathbb{P}_{\kappa} \otimes d \theta^{(n)}$ for each $n \in \mathbb{N}$. From (2.7), we get

$$
L_{*}^{(n)}(d \theta(f, \cdot))=d \theta^{(n)}(f, \cdot), \quad \forall f \in \Sigma, n \in \mathbb{N} .
$$

We assign $\Sigma$ the topology of locally uniform convergence. It suffices to show that $\mathcal{K}_{d \theta^{(n)}}\left(\mathbb{P}_{\kappa}\right) \widehat{\oplus} \mathbb{P}_{\kappa}$ and $\mathbb{P}_{\kappa} \otimes d \theta^{(n)}$ converge weakly to $\mathcal{K}_{d \theta}\left(\mathbb{P}_{\kappa}\right) \widehat{\oplus} \mathbb{P}_{\kappa}$ and $\mathbb{P}_{\kappa} \otimes d \theta$, respectively. To prove that $\mathcal{K}_{d \theta^{(n)}}\left(\mathbb{P}_{\kappa}\right) \widehat{\oplus} \mathbb{P}_{\kappa} \rightarrow \mathcal{K}_{d \theta}\left(\mathbb{P}_{\kappa}\right) \widehat{\oplus} \mathbb{P}_{\kappa}$, we define $\Sigma \times(0, \infty)$-valued random variables $h^{(n)}$ and $h$ on a probability space $(\Omega, \mathbb{P})$ such that their laws are the above measures, and a.s. $h^{(n)} \rightarrow h$. For this purpose, we choose $\Omega=\left(\Sigma_{\infty} \times(0, \infty)\right) \times \Sigma_{\oplus}, \mathbb{P}=\left(\mathbb{P}_{\kappa} \otimes d \theta\right) \times \mathbb{P}_{\kappa}$, $h((f, t), g)=\left(\mathcal{K}_{t}(f) \oplus g, t\right)$, and $h^{(n)}(f, t, g)=h\left(\left(f, L^{(n)}(t)\right), g\right)$. Using (2.8) and the convergence $L^{(n)}(t) \uparrow t$ it is easy to check that $h^{(n)}$ and $h$ satisfy the desired properties. To prove that $\mathbb{P}_{\kappa} \otimes d \theta^{(n)} \rightarrow \mathbb{P}_{\kappa} \otimes d \theta$, we choose $\Omega=\Sigma \times(0, \infty), \mathbb{P}=\mathbb{P}_{\kappa} \otimes d \theta, h=\mathrm{id}_{\Omega}$, and $h^{(n)}(f, t)=\left(f, L^{(n)}(t)\right)$. Then a.s. $h^{(n)} \rightarrow h$, and from (2.8) we see that the laws of $h^{(n)}$ and $h$ are $\mathbb{P}_{\kappa} \otimes d \theta^{(n)}$ and $\mathbb{P}_{\kappa} \otimes d \theta$, respectively. So we get (2.6) in the general case.

The statement after (2.6) follows from projecting both sides of (2.6) to $\Sigma$. 


\section{Schramm-Loewner Evolution}

In this section, we review the Loewner equations and the Schramm-Loewner Evolution (SLE). See [11, 24] for more details. We focus on chordal SLE, and will often omit the word "chordal" before "Loewner equation" or "SLE" when there is no confusion.

The definition of SLE uses the Loewner equations. Let's first review the (chordal) Loewner equation. Let $\lambda \in C([0, T))$, where $T \in(0, \infty]$. The Loewner equation driven by $\lambda$ is the following differential equation in the complex plane:

$$
\partial g_{t}(z)=\frac{2}{g_{t}(z)-\lambda(t)}, \quad 0 \leq t<T ; \quad g_{0}(z)=z .
$$

Let $\mathbb{H}=\{z \in \mathbb{C}: \operatorname{Im} z>0\}$. For $0 \leq t<T$, let $K_{t}$ denote the set of $z \in \mathbb{H}$ such that the solution $s \mapsto g_{s}(z)$ blows up before or at $t$. It turns out that $g_{t}$ maps $\mathbb{H} \backslash K_{t}$ conformally onto $\mathbb{H}$, and satisfies $g_{t}(z)=z+\frac{2 t}{z}+O\left(|z|^{-2}\right)$ as $z \rightarrow \infty$. We call $g_{t}$ and $K_{t}$ the Loewner maps and hulls, respectively, driven by $\lambda$.

Suppose for every $t \in[0, T), g_{t}^{-1}$ extends continuously from $\mathbb{H}$ to $\overline{\mathbb{H}}$. Throughout, we use $f_{t}$ to denote the continuation of $g_{t}^{-1}$ from $\overline{\mathbb{H}}$ into $\overline{\mathbb{H}}$. Also suppose that $\gamma(t):=f_{t}(\lambda(t))$, $0 \leq t<T$, is a continuous curve in $\overline{\mathbb{H}}$. Then we say that $\gamma$ is the Loewner curve driven by $\lambda$. In this case, for $0 \leq t<T, \mathbb{H} \backslash K_{t}$ is the unbounded connected component of $\mathbb{H} \backslash \gamma([0, t])$. The Loewner curve driven by $\lambda$ may not exist in general.

The Loewner equations satisfy the following scaling and translation properties. Suppose $\lambda(t), 0 \leq t<T$, generates Loewner maps $g_{t}$ and hulls $K_{t}, 0 \leq t<T$. Let $a>0$ and $b \in \mathbb{R}$, and $\lambda^{a, b}(t)=b+a \cdot \lambda\left(t / a^{2}\right), 0 \leq t<a^{2} T$. Then $\lambda^{a, b}$ generates the Loewner maps $z \mapsto b+a \cdot g_{t / a^{2}}((z-b) / a)$ and hulls $b+a \cdot K_{t / a^{2}}, 0 \leq t<a^{2} T$. If $\lambda$ generates a Loewner curve $\gamma$, then $\lambda^{a, b}$ also generates a Loewner curve, which is $b+a \cdot \gamma\left(\cdot / a^{2}\right)$.

Another simple and useful property of the Loewner equations is the renewal property. Suppose $\lambda(t), 0 \leq t<T$, generates Loewner maps $g_{t}$ and hulls $K_{t}, 0 \leq t<T$. Let $\tau \in[0, T)$. Then $\lambda(\tau+t), 0 \leq t<T-\tau$, generates Loewner maps $g_{\tau+t} \circ g_{\tau}^{-1}$ and hulls $g_{\tau}\left(K_{\tau+t} \backslash K_{\tau}\right)$, $0 \leq t<T-\tau$. If $\lambda$ and $\lambda(\tau+\cdot)$ generate Loewner curves $\gamma$ and $\gamma_{\tau}$, respectively, then $\gamma(\tau+t)=f_{\tau}\left(\gamma_{\tau}(t)\right), 0 \leq t<T-\tau$.

Let $\Sigma^{\mathbb{C}}$ denote the counterpart of $\Sigma$ with real valued continuous functions replaced by complex valued continuous functions. Let $\Sigma^{\mathcal{L}}$ denote the set of driving functions $\lambda \in \Sigma$ that generate a Loewner curve $\gamma$. Then $\Sigma^{\mathcal{L}} \in \mathcal{F}^{0}$, and the Loewner map $\mathcal{L}: \lambda \mapsto \gamma$ from $\Sigma^{\mathcal{L}}$ to $\Sigma^{\mathbb{C}}$ is measurable. We also define here the extended Loewner map $\widehat{\mathcal{L}}$ from $\left\{(\lambda, t): \lambda \in \Sigma^{\mathcal{L}}, 0 \leq t<T_{\lambda}\right\}$ to $\Sigma^{\mathbb{C}} \times \overline{\mathbb{H}}$ such that $\widehat{\mathcal{L}}(\lambda, t)=(\mathcal{L}(\lambda), \mathcal{L}(\lambda)(t))$.

Fix $\kappa>0$. Let $B(t), 0 \leq t<\infty$, be a standard Brownian motion. The $\mathrm{SLE}_{\kappa}$ process is defined by taking $\lambda(t)=\sqrt{\kappa} B(t), 0 \leq t<\infty$, in the Loewner equation. In this case, the Loewner curve $\gamma$ driven by $\lambda$ a.s. exists, and satisfies $\gamma(0)=0$ and $\lim _{t \rightarrow \infty} \gamma(t)=\infty$. Such $\gamma$ is called a standard $\mathrm{SLE}_{\kappa}$ curve (in $\mathbb{H}$ from 0 to $\infty$ ). In terms of measures, this means that $\mathbb{P}_{\kappa}$ (the law of $\left(\sqrt{\kappa} B_{t}\right)$ ) is supported by $\Sigma^{\mathcal{L}}$. The pushforward measure $\mathcal{L}_{*}\left(\mathbb{P}_{\kappa}\right)$ is then the law of a standard $\mathrm{SLE}_{\kappa}$ curve.

The scaling property of Loewner equations and the scaling property of Brownian motions together imply the scaling property of the SLE curve: if $\gamma$ is a standard SLE $_{\kappa}$ curve, then $t \mapsto a \gamma\left(t / a^{2}\right)$ is also a standard $\mathrm{SLE}_{\kappa}$ curve. The renewal and translation properties of Loewner equations and the strong Markov property of Brownian motions together imply the domain Markov property of SLE: if $\gamma$ is a standard $\mathrm{SLE}_{\kappa}$ curve, and $\tau$ is a finite 
stopping time, then conditioned on $\gamma(t), t \leq \tau$, there is a standard $\operatorname{SLE}_{\kappa}$ curve $\widehat{\gamma}$ such that $\gamma(\tau+t)=f_{\tau}\left(\widehat{\gamma}(t)+\lambda_{\tau}\right), t \geq 0$.

The definition of $\mathrm{SLE}_{\kappa}$ extends to other simply connected domains by conformal maps. Let $D$ be a simply connected domain with locally connected boundary. Let $a$ and $b$ be two distinct prime ends ([1]) of $D$. Let $f$ be a conformal map from $\mathbb{H}$ onto $D$ such that $f(0)=a$ and $f(\infty)=b$. Let $\gamma$ be a standard $\operatorname{SLE}_{\kappa}$ curve. Then $f \circ \gamma$ is called an $\operatorname{SLE}_{\kappa}$ curve in $D$ from $a$ to $b$. The local connectedness of $\partial D$ is used to guarantee that $f$ extends continuously to $\overline{\mathbb{H}}$ so that $f \circ \gamma$ is a continuous curve in $\bar{D}$. This condition may be weakened in some cases. Although the $f$ is not unique, the law of $f \circ \gamma$ is unique up to a linear time-change, thanks to the scaling property of a standard $\mathrm{SLE}_{\kappa}$ curve. From now on, an $\mathrm{SLE}_{\kappa}$ curve without the domain and two prime ends specified is always a standard $\mathrm{SLE}_{\kappa}$ curve, and the word "standard" will often be omitted

The behavior of an $\mathrm{SLE}_{\kappa}$ curve depends on the value of $\kappa$. Let $\gamma$ be an $\operatorname{SLE}_{\kappa}$ curve. If $\kappa \in(0,4]$, then $\gamma$ is a simple curve, and does not intersect $\mathbb{R}$ after the time 0 ; if $\kappa>4$, then $\gamma$ will intersect itself and $\mathbb{R}$ after the time 0 . If $\kappa \geq 8, \gamma$ is space-filling: it visits every point in $\overline{\mathbb{H}}$; if $\kappa<8$, then for every $z_{0} \in \overline{\mathbb{H}} \backslash\{0\}$, the probability that $\gamma$ visits $z_{0}$ is 0 . Moreover, the Hausdorff dimension of $\gamma$ is $\min \left\{1+\frac{\kappa}{8}, 2\right\}$ (cf. [4]).

$\operatorname{SLE}_{\kappa}(\rho)$ is a variant of $\mathrm{SLE}_{\kappa}$. Its definition involves one or more force points, which lie on the boundary or in the interior of the domain. For the purpose here, we consider the case that there is only one force point, which is an interior point. Let $\rho \in \mathbb{R}, a_{0} \in \mathbb{R}$ and $z_{0} \in \mathbb{H}$. An $\operatorname{SLE}_{\kappa}(\rho)$ process started from $a_{0} \in \mathbb{R}$ with force point at $z_{0} \in \mathbb{H}$ is the solution of the Loewner equation driven by $\lambda_{t}, 0 \leq t<T_{z_{0}}$, which is the solution of the SDE:

$$
d \lambda_{t}=\sqrt{\kappa} d B_{t}+\operatorname{Re} \frac{\rho}{\lambda_{t}-g_{t}^{\lambda}\left(z_{0}\right)} d t, \quad \lambda_{0}=a_{0} .
$$

Here $g_{t}^{\lambda}$ are the Loewner maps driven by $\lambda$, and $\left[0, T_{z_{0}}\right)$ is the maximal solution interval. Let $\mathbb{P}_{z_{0}}^{\kappa, \rho}$ denote the law of $\lambda_{t}, 0 \leq t<T_{z_{0}}$. From Proposition 2.3. we know that $\mathbb{P}_{z_{0}}^{\kappa, \rho} \triangleleft \mathbb{P}_{\kappa}$. Thus, $\mathbb{P}_{z_{0}}^{\kappa, \rho}$ is supported by $\Sigma^{\mathcal{L}}$, i.e., the Loewner curve $\gamma$ driven by $\lambda$ a.s. exists, which is called a (standard) $\operatorname{SLE}_{\kappa}(\rho)$ curve started from $a_{0}$ with force point at $z_{0}$. The following proposition is a result of 22 .

Proposition 3.1. For any $\kappa>0$ and $\rho \leq \frac{\kappa}{2}-4$,

(i) a.s. $T_{z_{0}}<\infty$ and $\lim _{t \rightarrow T_{z_{0}}^{-}} \lambda_{t} \in \mathbb{R}$;

(ii) a.s. $\lim _{t \rightarrow T_{z_{0}}^{-}} \gamma(t)=z_{0}$.

From the above proposition we see that, if $\rho \leq \frac{\kappa}{2}-4$, then $\mathbb{P}_{z_{0}}^{\kappa, \rho}$ is supported by $\Sigma^{\oplus}$, and we may define $\mathbb{P}_{z_{0}}^{\kappa, \rho} \oplus \mathbb{P}_{\kappa}$, which is supported by $\Sigma_{\mathcal{L}}$. The pushforward measure $\mathcal{L}_{*}\left(\mathbb{P}_{z_{0}}^{\kappa, \rho} \oplus \mathbb{P}_{\kappa}\right)$ is called the law of a standard extended $\operatorname{SLE}_{\kappa}(\rho)$ curve through $z_{0}$, which is supported by the continuous curves in $\overline{\mathbb{H}}$ from 0 to $\infty$ that pass through $z_{0}$. In other words, a standard extended $\operatorname{SLE}_{\kappa}(\rho)$ curve through $z_{0}$ is defined by continuing an $\operatorname{SLE}_{\kappa}(\rho)$ curve started from 0 with force point at $z_{0}$ by an $\mathrm{SLE}_{\kappa}$ curve in the remaining domain from $z_{0}$ to $\infty$.

Using conformal maps, we may define an extended $\operatorname{SLE}_{\kappa}(\rho)$ curve (for $\rho \leq \frac{\kappa}{2}-4$ ) in a simply connected domain from one prime end to another prime end through an interior point. We will mainly work on extended $\operatorname{SLE}_{\kappa}(\rho)$ curves in $\mathbb{H}$ from 0 to $\infty$ through some $z_{0} \in \mathbb{H}$, and so will omit the word "standard". 
We now derive the local Radon-Nikodym derivative of $\mathbb{P}_{z_{0}}^{\kappa, \rho}$ w.r.t. $\mathbb{P}_{\kappa}$. Let $\lambda_{t}=\sqrt{\kappa} B_{t}$, and $g_{t}$ be the Loewner maps driven by $\lambda$. Fix $z_{0}=x_{0}+i y_{0} \in \mathbb{H}$. For $0 \leq t<T_{z_{0}}$, let

$$
Z_{t}=g_{t}\left(z_{0}\right)-\lambda_{t}, \quad X_{t}=\operatorname{Re} Z_{t}, \quad Y_{t}=\operatorname{Im} Z_{t}, \quad D_{t}=\left|g_{t}^{\prime}\left(z_{0}\right)\right| .
$$

From Loewner's equation, we see that $Y_{t}$ and $D_{t}$ satisfy the ODEs:

$$
\frac{d Y_{t}}{Y_{t}}=\frac{-2}{X_{t}^{2}+Y_{t}^{2}} d t, \quad \frac{d D_{t}}{D_{t}}=\frac{-2\left(X_{t}^{2}-Y_{t}^{2}\right)}{\left(X_{t}^{2}+Y_{t}^{2}\right)^{2}} d t
$$

and $X_{t}$ satisfies the SDE

$$
d X_{t}=-\sqrt{\kappa} d B_{t}+\frac{2 X_{t}}{X_{t}^{2}+Y_{t}^{2}} d t
$$

Define

$$
M_{t}^{\kappa, \rho}\left(z_{0}\right)=\left|Z_{t}\right|^{\frac{\rho}{\kappa}} \cdot Y_{t}^{\frac{\rho^{2}}{8 \kappa}} \cdot D_{t}^{\frac{\rho}{\kappa}\left(1-\frac{\kappa}{4}+\frac{\rho}{8}\right)}, \quad 0 \leq t<T_{z_{0}}
$$

and

$$
G^{\kappa, \rho}\left(z_{0}\right)=M_{0}^{\kappa, \rho}\left(z_{0}\right)=\left|z_{0}\right|^{\frac{\rho}{\kappa}} \cdot \operatorname{Im}\left(z_{0}\right)^{\frac{\rho^{2}}{8 \kappa}} .
$$

Using Itô's formula and (3.2)-(3.3), it is straightforward to check that $\left(M_{t}^{\kappa, \rho}\left(z_{0}\right)\right)$ is an $\left(\mathcal{F}_{t}^{0}\right)$-adapted continuous local martingale, and satisfies the SDE:

$$
d M_{t}^{\kappa, \rho}\left(z_{0}\right)=M_{t}^{\kappa, \rho}\left(z_{0}\right) \cdot \operatorname{Re} \frac{\rho / \sqrt{\kappa}}{\lambda_{t}-g_{t}\left(z_{0}\right)} d B_{t}, \quad 0 \leq t<T_{z_{0}} .
$$

We further define

$$
M_{t}^{\kappa, \rho}\left(z_{0}\right)=0, \quad T_{z_{0}} \leq t<\infty .
$$

For any measurable subset $U$ of $\overline{\mathbb{H}}$, define

$$
\Psi_{t}^{\kappa, \rho}(U)=\int_{U \cap \mathbb{H}} M_{t}^{\kappa, \rho}(z) d A(z) .
$$

Throughout this paper, we use $d A$ to denote the Lebesgue measure on $\mathbb{C}$. Since $\left(M_{t}^{\kappa, \rho}(z)\right)$ is a positive local martingale, it is also a supermartingale. Thus, if $\int_{U} G^{\kappa, \rho}(z) d A(z)<\infty$, then $\left(\Psi_{t}^{\kappa, \rho}(U)\right)$ is also a supermartingale, which has to be $\mathbb{P}_{\kappa}$-a.s. finite.

From Proposition 2.3 we know that

$$
\frac{\left.d \mathbb{P}_{z_{0}}^{\kappa, \rho}\right|_{\mathcal{F}_{t}^{0} \cap \Sigma_{t}}}{\left.d \mathbb{P}_{\kappa}\right|_{\mathcal{F}_{t}^{0} \cap \Sigma_{t}}}=\frac{M_{t}^{\kappa, \rho}\left(z_{0}\right)}{G^{\kappa, \rho}\left(z_{0}\right)}, \quad 0 \leq t<\infty
$$

From Proposition 2.1. $(z, E) \mapsto \mathbb{P}_{z}^{\kappa, \rho}(E)$ is a probability kernel from $\mathbb{H}$ to $\left(\Sigma, \mathcal{F}^{0}\right)$. Thus, for any measurable subset $U$ of $\overline{\mathbb{H}}$, we may define the measure

$$
\mathbb{P}_{U}^{\kappa, \rho}=\int_{U \cap \mathbb{H}} \mathbb{P}_{z}^{\kappa, \rho} G^{\kappa, \rho}(z) d A(z) .
$$

Moreover, if $\int_{U} G^{\kappa, \rho}(z) d A(z)<\infty$, then $\mathbb{P}_{U}^{\kappa, \rho} \triangleleft \mathbb{P}_{\kappa}$, and

$$
\frac{\left.d \mathbb{P}_{U}^{\kappa, \rho}\right|_{\mathcal{F}_{t}^{0} \cap \Sigma_{t}}}{\left.d \mathbb{P}_{\kappa}\right|_{\mathcal{F}_{t}^{0} \cap \Sigma_{t}}}=\Psi_{t}^{\kappa, \rho}(U), \quad 0 \leq t<\infty
$$




\section{Natural Parametrization}

Fix $\kappa \in(0,8)$. Let $d=1+\frac{\kappa}{8}$ be the Hausdorff dimension of $\mathrm{SLE}_{\kappa}$ curves. We will review the natural parametrization of $\mathrm{SLE}_{\kappa}$ in this section.

First, we review the definition of the SLE Green's function. The Green's function for an $\mathrm{SLE}_{\kappa}$ curve $\gamma$ in a simply connected domain $D$ from one prime end $a$ to another prime end $b$ is

$$
G_{(D ; a, b)}(z):=\lim _{r \rightarrow 0^{+}} r^{d-2} \mathbb{P}[\operatorname{dist}(z, \gamma)<r], \quad z \in D,
$$

provided that the limit exists. It is clear that, if the $\mathrm{SLE}_{\kappa}$ Green's function exists for one configuration $(D ; a, b)$, then it exists for all configurations, and it satisfies the conformal covariance, i.e., if $f$ maps $(D ; a, b)$ conformally onto $\left(D^{\prime} ; a^{\prime}, b^{\prime}\right)$, then

$$
G_{(D ; a, b)}(z)=\left|f^{\prime}(z)\right|^{2-d} G_{\left(D^{\prime} ; a^{\prime}, b^{\prime}\right)}(f(z)) .
$$

It is proved in [14 that there is an unknown positive constant $C_{\kappa}>0$ depending only on $\kappa$ such that

$$
G_{(\mathbb{H} ; 0, \infty)}(z)=C_{\kappa}|z|^{d-2} \sin ^{\frac{\kappa}{8}+\frac{8}{\kappa}-2}(\arg z), \quad z \in \mathbb{H},
$$

We will write $G(z)$ for $G_{(\mathbb{H} ; 0, \infty)}(z)$. Define

$$
M_{t}(z)=\left|g_{t}^{\prime}(z)\right|^{2-d} G\left(g_{t}(z)-\lambda(t)\right), \quad 0 \leq t<T_{z} .
$$

and $M_{t}(z)=0$ for $t \geq T_{z}$. For any measurable set $U \subset \overline{\mathbb{H}}$, define $\Psi_{t}(U)=\int_{U \cap \mathbb{H}} M_{t}(z) d A(z)$. It is easy to check that $G(z), M_{t}(z), \Psi_{t}(U)$ agree with $C_{\kappa}$ times $G^{\kappa, \kappa-8}(z), M_{t}^{\kappa, \kappa-8}(z)$, $\Psi_{t}^{\kappa, \kappa-8}(U)$, respectively, defined by (3.4)-(3.7). From (3.8) we see that locally weighting $\sqrt{\kappa} B_{t}$ using $\left(M_{t}\left(z_{0}\right) / G\left(z_{0}\right)\right)$ generates a driving $\operatorname{SLE}_{\kappa}(\kappa-8)$ process with force point at $z_{0}$.

A two-sided radial $\mathrm{SLE}_{\kappa}$ curve is just an extended $\operatorname{SLE}_{\kappa}(\kappa-8)$ curve. This means that the law of a (standard) two-sided radial $\mathrm{SLE}_{\kappa}$ curve through $z_{0} \in \mathbb{H}$ can be expressed by $\mathcal{L}_{*}\left(\mathbb{P}_{z_{0}}^{\kappa, \kappa-8} \oplus \mathbb{P}_{\kappa}\right)$. Two-sided radial SLE is important because it can be understood as an $\mathrm{SLE}_{\kappa}$ curve conditioned to pass though an interior point. To make this rigorous, one may condition an $\operatorname{SLE}_{\kappa}$ curve $\gamma$ on the event that $\operatorname{dist}\left(z_{0}, \gamma\right)<r$ for a fixed interior point $z_{0}$, and then pass to the limit $r \rightarrow 0$.

From the reversibility of chordal $\mathrm{SLE}_{\kappa}$ (cf. 29, 21) it is easy to see that two-sided radial $\mathrm{SLE}_{\kappa}$ also satisfies reversibility, i.e., the time-reversal of a two-sided radial $\mathrm{SLE}_{\kappa}$ curve in a simply connected domain $D$ from $a$ to $b$ through $z_{0}$ agrees with a two-sided radial $\mathrm{SLE}_{\kappa}$ curve in $D$ from $b$ to $a$ through $z_{0}$, up to a reparametrization. This property is not satisfied by extended $\operatorname{SLE}_{\kappa}(\rho)$ processes for $\rho \neq \kappa-8$.

Recall that $\mathbb{P}^{\kappa, \kappa-8}$ is a kernel from $\mathbb{H}$ to $\left(\Sigma, \mathcal{F}^{0}\right)$. So we can defined the measure $\mathbb{P}_{U}=$ $\int_{U \cap \mathbb{H}} \mathbb{P}_{t}^{\kappa, \kappa-8}(z) G(z) d A(z)$ for any measurable set $U \subset \overline{\mathbb{H}}$. Then $\mathbb{P}_{U}$ equals $C_{\kappa}$ times the $\mathbb{P}_{U}^{\kappa, \kappa-8}$ defined by (3.9). From (3.10), if $\int_{U} G(z) d A(z)<\infty$, then $\mathbb{P}_{U} \triangleleft \mathbb{P}_{\kappa}$ and

$$
\frac{\left.d \mathbb{P}_{U}\right|_{\mathcal{F}_{t}^{0} \cap \Sigma_{t}}}{\left.d \mathbb{P}_{\kappa}\right|_{\mathcal{F}_{t}^{0} \cap \Sigma_{t}}}=\Psi_{t}(U), \quad 0 \leq t<\infty
$$

It is proved by Lawler and Zhou (20]) that, if $U$ is a pre-compact measurable subset of $\mathbb{H}$, i.e., $\bar{U}$ is a compact subset of $\mathbb{H}$, then $\left(\Psi_{t}(U)\right)$ is of class $\mathcal{D}$, i.e., $\left\{\Psi_{T}(U)\right.$ : $T$ is a finite stopping time $\}$ is uniformly integrable. In this case they can apply the DoobMeyer decomposition theorem to get a unique continuous increasing $\left(\mathcal{F}_{t}^{B}\right)$-adapted process 
$\Theta_{t}(U)$ such that $\Theta_{0}(U)=0$ and $M_{t}(U):=\Psi_{t}(U)+\Theta_{t}(U)$ is a uniformly integrable $\left(\mathcal{F}_{t}^{B}\right)$ martingale. This means that $M_{\infty}(U):=\lim _{t \rightarrow \infty} M_{t}(U)$ a.s. exists, and $\mathbb{E}_{\kappa}\left[M_{\infty}(U) \mid \mathcal{F}_{t}^{B}\right]=$ $M_{t}(U), 0 \leq t<\infty$. From Lemma A.2, we know that $\Psi_{\infty}(U)=0$. Thus,

$$
\mathbb{E}\left[\Theta_{\infty}(U) \mid \mathcal{F}_{t}^{B}\right]-\Theta_{t}(U)=\Psi_{t}(U), \quad 0 \leq t<\infty .
$$

The process $\left(\Theta_{t}(U)\right)$ determines a $\mathbb{P}_{\kappa}$-kernel $d \Theta(U)$. Then we may define the killing $\mathcal{K}_{d \Theta(U)}\left(\mathbb{P}_{\kappa}\right)$. From (2.1), we have

$$
\mathbb{P}_{\kappa}-\text { a.s. }, \quad \frac{\left.d \mathcal{K}_{d \Theta(U)}\left(\mathbb{P}_{\kappa}\right)\right|_{\mathcal{F}_{t}^{0} \cap \Sigma_{t}}}{\left.d \mathbb{P}_{\kappa}\right|_{\mathcal{F}_{t}^{0} \cap \Sigma_{t}}}=\mathbb{E}\left[\Theta_{\infty}(U) \mid \mathcal{F}_{t}^{B}\right]-\Theta_{t}(U), \quad 0 \leq t<\infty .
$$

Combining (4.2), (4.3) and (4.4), we see that,

$$
\mathbb{P}_{U}=\mathcal{K}_{d \Theta(U)}\left(\mathbb{P}_{\kappa}\right)
$$

It is easy to check that, if $U_{1}$ and $U_{2}$ are disjoint pre-compact measurable subsets of $\mathbb{H}$, then a.s. $\Theta_{t}\left(U_{1} \cup U_{2}\right)=\Theta_{t}\left(U_{1}\right)+\Theta_{t}\left(U_{2}\right)$ for $0 \leq t<\infty$. Thus, the kernel $d \Theta_{t}(U)$ (and also $\Theta_{t}(U)$ ) is increasing in $U$. We may extend the definition of $\Theta_{t}(U)$ to any measurable subset $U$ of $\overline{\mathbb{H}}$ as follows. Let $\left(U_{n}\right)$ be a sequence of compact subsets of $\mathbb{H}$ such that $U_{n}$ is contained in the interior of $U_{n+1}$ for every $n \in \mathbb{N}$, and $\mathbb{H}=\bigcup U_{n}$. For any measurable subset $U$ of $\overline{\mathbb{H}}$, we first define the kernel $\mu_{U}=\lim _{n \rightarrow \infty} d \Theta\left(U \cap U_{n}\right)$, then let $\Theta_{t}(U)=\mu_{U}([0, t]), t \geq 0$ (so $\left.d \Theta(U)=\mu_{U}\right)$. The definition does not depend on the choice of $\left(U_{n}\right)$. In particular, $\Theta_{t}:=\Theta_{t}(\overline{\mathbb{H}})$ is called the natural parametrization of $\gamma$.

The $\Theta_{t}(U)$ is understood as the total time that the chordal $\operatorname{SLE}_{\kappa}$ curve $\gamma$ spends in $U$ in the natural parametrization before time $t$. Define the kernel $\mathcal{M}_{U}$ from $\mathcal{L}\left(\Sigma^{\mathcal{L}}\right)$ to $\overline{\mathbb{H}}$ by

$$
\mathcal{M}_{U}(\gamma, \cdot):=\gamma_{*}(d \Theta(U))
$$

This is the $d$-dimensional Minkowski content measure of $\gamma$ in $U$.

Here the Minkowski content measure of a set $S$ in a domain $U$ is a measure, say $\mathcal{M}$ supported by $S \cap U$, which satisfies the property that for any compact set $K \subset U, K \cap S$ has Minkowski content, which equals to $\mathcal{M}(K)$ and is finite. It satisfies the property of conformal covariance. From the work of [14, we know that an $\operatorname{SLE}_{\kappa}$ curve for $\kappa \in(0,8)$ in $\mathbb{H}$ from 0 to $\infty$ possesses $\left(1+\frac{\kappa}{8}\right)$-dimensional Minkowski content measure, which equals to the pushforward measure of the curve function of the natural parametrization (as a measure on the time interval). Using conformal covariance, we then know that any chordal $\mathrm{SLE}_{\kappa}$ curve possesses Minkowski content measure in the domain that it is defined. The reader is referred to [33, Section 2.3] for details of the notation.

Theorem 4.1. Let $\kappa \in(0,8)$. Let $U$ be any measurable subset of $\overline{\mathbb{H}}$. Then we have

$$
\begin{gathered}
\mathbb{P}_{U} \widehat{\oplus} \mathbb{P}_{\kappa}=\mathbb{P}_{\kappa} \otimes d \Theta(U), \\
\mathcal{L}_{*}\left(\mathbb{P}_{z}^{\kappa, \kappa-8} \oplus \mathbb{P}_{\kappa}\right) \overleftarrow{\otimes} \mathbf{1}_{U} G(z) d A(z)=\mathcal{L}_{*}\left(\mathbb{P}_{\kappa}\right) \otimes \mathcal{M}_{U},
\end{gathered}
$$

where $\mathcal{M}_{U}$ is the d-dimensional Minkowski content measure of $\gamma \cap U$.

Proof. If $U$ is pre-compact in $\mathbb{H}$, then (4.7) follows from (4.5) and Proposition 2.4. For general measurable $U \subset \mathbb{H}$, (4.7) follows from the above special case and a limiting procedure. 
We now apply the extended Loewner map $\widehat{\mathcal{L}}(\lambda, t)=(\mathcal{L}(\lambda), \mathcal{L}(\lambda)(t))$ to (4.7). We observe that $\widehat{\mathcal{L}}_{*}\left(\mathbb{P}_{z}^{\kappa, \kappa-8} \widehat{\oplus} \mathbb{P}_{\kappa}\right)=\mathcal{L}_{*}\left(\mathbb{P}_{z}^{\kappa, \kappa-8} \oplus \mathbb{P}_{\kappa}\right) \otimes \delta_{z}$, where $\delta_{z}$ is the Dirac measure at $z$. Integrating the equality over $z$ against the measure $\mathbf{1}_{U} G(z) d A(z)=\mathbf{1}_{U \cap \mathbb{H}} G(z) d A(z)$, we get

$$
\widehat{\mathcal{L}}_{*}\left(\mathbb{P}_{U}^{\kappa, \kappa-8} \widehat{\oplus} \mathbb{P}_{\kappa}\right)=\mathcal{L}_{*}\left(\mathbb{P}_{z}^{\kappa, \kappa-8} \oplus \mathbb{P}_{\kappa}\right) \overleftarrow{\otimes} \mathbf{1}_{U} G(z) d A(z)
$$

Using (4.6) and the definition of $\widehat{\mathcal{L}}$, we find that

$$
\widehat{\mathcal{L}}_{*}\left(\mathbb{P}_{\kappa} \otimes d \Theta(U)\right)=\mathcal{L}_{*}\left(\mathbb{P}_{\kappa}\right) \otimes \mathcal{M}_{U}
$$

Then (4.7), (4.9) and (4.10) together imply (4.8).

Corollary 4.1. Let $\kappa \in(0,8)$. Let $U$ be a measurable subset of $\mathbb{H}$ with $\int_{U} G(z) d A(z)<\infty$. If we integrate the laws of two-sided radial $S L E_{\kappa}$ curves through $z$ against the measure $\mathbf{1}_{U} G(z) d A(z)$, then we get a finite measure on curves, which is absolutely continuous w.r.t. the law of $S L E_{\kappa}$ curve, and the Radon-Nikodym derivative is the d-dimensional Minkowski content of $\gamma \cap U$.

Proof. This follows from projecting both sides of (4.8) to $\Sigma^{\mathbb{C}}$ and that $\mathbb{E}_{\kappa}\left[\mathcal{M}_{U}(\gamma, \cdot)\right]=$ $\mathbb{E}_{\kappa}\left[\Theta_{\infty}(U)\right]=\int_{U} G(z) d A(z)<\infty$.

Corollary 4.2. Let $\kappa \in(0,8)$. Suppose $(\gamma, z)$ is a $C([0, \infty), \mathbb{C}) \times \overline{\mathbb{H}}$-valued random variable with the properties that $\gamma$ has the law of an $S L E_{\kappa}$ curve, and given $\gamma$, the law of $z$ is absolutely continuous w.r.t. the d-dimensional Minkowski content measure of $\gamma$. Then the law of $z$ is absolutely continuous w.r.t. $\mathbf{1}_{\mathbb{H}} d A(z)$, and the law of $\gamma$ given $z$ is absolutely continuous w.r.t. the law of a two-sided radial $S L E_{\kappa}$ curve through $z$.

Proof. From the assumption, we see that the law of $(\gamma, z)$ is absolutely continuous w.r.t. the measure in either side of (4.8) with $U=\mathbb{H}$.

\section{Remarks.}

1. Roughly speaking, the meaning of (4.8) is that the following two methods generate the same measure on the space of curve-point pairs:

(i) first sample a point according to the measure $\mathbf{1}_{U} G(z) d A(z)$, and then sample a two-sided radial $\mathrm{SLE}_{\kappa}$ curve $\gamma$ through $z$;

(ii) first sample an $\mathrm{SLE}_{\kappa}$ curve $\gamma$, and then sample a point $z$ on $\gamma$ according to the $d$-dimensional Minkowski content measure of $\gamma \cap U$. Here the measure of $\gamma$ is changed after sampling $z$ because $\mathcal{M}_{U}$ is not a probability kernel.

2. Corollary 4.2 means that, if we sample a random point $z$ on a chordal $\operatorname{SLE}_{\kappa}$ curve $\gamma$ according to a law that is absolutely continuous w.r.t. the $d$-dimensional Minkowski content measure, and then condition on $z$, then the curve $\gamma$ looks "similar" to a twosided radial $\mathrm{SLE}_{\kappa}$ curve through $z$.

Corollary 4.3. Let $D$ be a simply connected domain with two distinct prime ends a and $b$. Let $\mathbb{P}_{D ; a, b}^{\kappa}$ denote the law of an $S L E_{\kappa}$ curve in $D$ from a to $b$. For any $z \in D$, let $\mathbb{P}_{D ; a, b ; z}^{\kappa}$ denote the law of a two-sided radial $S L E_{\kappa}$ curve in $D$ from a to $b$ through $z$. For any measurable set $U \subset D$, let $\mathcal{M}_{U}(\gamma, \cdot)$ be the d-dimensional Minkowski content measure on $\gamma \cap U$. Then

$$
\mathbb{P}_{D ; a, b ; z}^{\kappa} \overleftarrow{\otimes} \mathbf{1}_{U} G_{D ; a, b}(z) d A(z)=\mathbb{P}_{D ; a, b}^{\kappa} \otimes \mathcal{M}_{U}
$$


Moreover, if $\int_{U} G_{D ; a, b}(z) d A(z)<\infty$, then $\int_{U} \mathbb{P}_{D ; a, b ; z}^{\kappa} d A(z) \ll \mathbb{P}_{D ; a, b}^{\kappa}$, and the RadonNikodym derivative is the d-dimensional Minkowski content of $\gamma \cap U$.

Proof. Formula (4.11) follows immediately from (4.8), the conformal covariance of SLE $\mathrm{K}_{\kappa}$ Green's function, and the transformation rule of $d$-dimensional Minkowski content measure under confomal maps. The rest follows from projecting the measures in (4.11) to $\Sigma^{\mathbb{C}}$.

Remark. The above corollary in the case $\kappa \leq 4$ was proved earlier by Laurie Field in [8], where he assumed that the domain $D$ is bounded and has analytic boundary.

Inspired by Theorem 4.1 and its corollaries, we make the following definition.

Definition 4.1. Let $\kappa>0$ and $\rho \leq \frac{\kappa}{2}-4$. We say that $S L E_{\kappa}$ admits an $S L E_{\kappa}(\rho)$ decomposition if (4.7) holds with $G(z)$ replaced by $G^{\kappa, \rho}(z)$ and $\mathbb{P}_{U}$ replaced by $\mathbb{P}_{U}^{\kappa, \rho}$, for some increasing adapted process $\Theta_{t}(U)$, which then implies 4.8$)$ with $\mathbb{P}_{z}^{\kappa, \kappa-8}$ replaced by $\mathbb{P}_{z}^{\kappa, \rho}$ and $\mathcal{M}_{U}$ defined by (4.6) for the new $\Theta_{t}(U)$.

We have shown that $\operatorname{SLE}_{\kappa}$ admits an $\operatorname{SLE}_{\kappa}(\kappa-8)$ decomposition for $\kappa \in(0,8)$. From the argument, we see that, one approach to show that $\operatorname{SLE}_{\kappa}$ admits an $\operatorname{SLE}_{\kappa}(\rho)$ decomposition is to show that for any pre-compact measurable subset $U$ of $\mathbb{H}, \Psi_{t}^{\kappa, \rho}(U)$ defined in (3.7) is of class $\mathcal{D}$, and then apply the Doob-Meyer decomposition theorem. But this is very difficult in general (cf. [15, 20]). In the next section, we will show that $\operatorname{SLE}_{\kappa}$ admits an $\operatorname{SLE}_{\kappa}(-8)$ decomposition for any $\kappa>0$ using a different approach.

\section{Capacity Parametrization}

Fix $\kappa>0$. We say that a chordal $\mathrm{SLE}_{\kappa}$ curve $\gamma$ (with the reparametrization given by its definition) has capacity parametrization because the hull $K_{t}$ determined by $\gamma([0, t])$ has half-plane capacity $2 t$ for each $t \geq 0$. In this section we consider $\operatorname{SLE}_{\kappa}(\rho)$ processes with $\rho=-8$, which turns out to be closely related to the capacity parametrization.

For any $z \in \mathbb{H}$ and $N>0$, let $\mathbb{P}_{z ; N}^{\kappa,-8}$ be the measure on $\left(\Sigma, \mathcal{F}^{0}\right)$ defined by $\mathbb{P}_{z ; N}^{\kappa,-8}(E)=$ $\mathbb{P}_{z}^{\kappa,-8}\left(E \backslash \Sigma_{N}\right)$. Note that $\mathbb{P}_{z ; N}^{\kappa,-8}$ is not a probability measure. Then we have $\mathbb{P}_{z ; N}^{\kappa,-8} \ll \mathbb{P}_{z}^{\kappa,-8}$, and so $\mathbb{P}_{z: N}^{\kappa,-8} \triangleleft \mathbb{P}_{z}^{\kappa,-8}$. Since $\mathbb{P}_{z}^{\kappa,-8} \triangleleft \mathbb{P}_{\kappa}$, we get $\mathbb{P}_{z ; N}^{\kappa,-8} \triangleleft \mathbb{P}_{\kappa}$. We now calculate the local Radon-Nikodym derivatives of $\mathbb{P}_{z ; N}^{\kappa,-8}$ w.r.t. $\mathbb{P}_{\kappa}$.

Let $\lambda_{t}=\sqrt{\kappa} B_{t}$, and $g_{t}$ the Loewner maps driven by $\lambda$. Let $M_{t}(z)=M_{t}^{\kappa,-8}(z)$ and $G(z)=G^{\kappa,-8}(z)$ be as defined by (3.4)-(3.6). Then we have $G(z)=(\operatorname{Im} z /|z|)^{8 / \kappa} ; M_{t}(z)=$ $G\left(g_{t}(z)-\lambda_{t}\right)\left|g_{t}^{\prime}(z)\right|^{2}, 0 \leq t<T_{z} ;$ and $M_{t}(z)=0, T_{z} \leq t<\infty$.

Let $t \in[0, \infty)$ and $E \in \mathcal{F}_{t}^{0} \cap \Sigma_{t}$. If $t \geq N$, then $\mathbb{P}_{z ; N}^{\bar{\kappa},-8}(E)=0$ because $E \subset \Sigma_{t} \subset \Sigma_{N}$. If $t<N$, from (3.8), we get

$$
\begin{gathered}
\mathbb{P}_{z ; N}^{\kappa,-8}(E)=\mathbb{P}_{z}^{\kappa,-8}\left(E \backslash \Sigma_{N}\right)=\mathbb{P}_{z}^{\kappa,-8}(E)-\mathbb{P}_{z}^{\kappa,-8}\left(E \cap \Sigma_{N}\right) \\
=\int_{E} \frac{M_{t}(z)}{G(z)} d \mathbb{P}_{\kappa}-\int_{E \cap \Sigma_{N}} \frac{M_{N}(z)}{G(z)} d \mathbb{P}_{\kappa}=\frac{1}{G(z)} \int_{E}\left(M_{t}(z)-\mathbb{E}_{\kappa}\left[M_{N}(z) \mid \mathcal{F}_{t}^{0}\right]\right) d \mathbb{P}_{\kappa} .
\end{gathered}
$$

Here the third "=" holds because $E \in \mathcal{F}_{t}^{0} \cap \Sigma_{t}$ and $E \cap \Sigma_{N} \in \mathcal{F}_{N}^{0} \cap \Sigma_{N}$, and the fourth "=" holds because $\mathbb{P}_{\kappa}$ is supported by $\Sigma_{\infty} \subset \Sigma_{N}$. Thus,

$$
\frac{\left.d \mathbb{P}_{z ; N}^{\kappa,-8}\right|_{\mathcal{F}_{t}^{0} \cap \Sigma_{t}}}{\left.d \mathbb{P}_{\kappa}\right|_{\mathcal{F}_{t}^{0} \cap \Sigma_{t}}}= \begin{cases}\frac{1}{G(z)}\left(M_{t}(z)-\mathbb{E}_{\kappa}\left[M_{N}(z) \mid \mathcal{F}_{t}^{0}\right]\right), & 0 \leq t<N ; \\ 0, & N \leq t<\infty .\end{cases}
$$


For $t \geq 0$, define $G_{t}$ on $\mathbb{H}$ by

$$
G_{t}(z)=M_{0}(z)-\mathbb{E}_{\kappa}\left[M_{t}(z)\right]=G(z)-\mathbb{E}_{\kappa}\left[M_{t}(z)\right]
$$

and let $C_{\kappa, t}=\int_{\mathbb{H}} G_{t}(z) d A(z)$. From (3.8), we get

$$
\frac{G_{t}(z)}{G(z)}=1-\int_{\Sigma_{t}} \frac{M_{t}(z)}{G(z)} d \mathbb{P}_{\kappa}=1-\mathbb{P}_{z}^{\kappa, \rho}\left[\Sigma_{t}\right]=\mathbb{P}_{z}^{\kappa, \rho}\left[T_{z} \leq t\right]
$$

Thus, $G_{0} \equiv 0, G_{t} \geq 0$ for $t>0$, and

$$
\lim _{t \rightarrow \infty} G_{t}(z)=G(z) \mathbb{P}_{z}^{\kappa, \rho}\left[T_{z}<\infty\right]=G(z)
$$

From the scaling property of $\operatorname{SLE}_{\kappa}(-8)$ process, we get $\frac{G_{a a_{t}}(a z)}{G(a z)}=\frac{G_{t}(z)}{G(z)}$ for any $a>0$. Since $G(a z)=G(z)$, we get $G_{a^{2} t}(a z)=G_{t}(z)$. Especially, we have

$$
G_{t}(z)=G_{1}\left(\frac{z}{\sqrt{t}}\right), \quad t>0 .
$$

Lemma 5.1. We have $C_{\kappa, t}=t C_{\kappa, 1}$ for every $t \geq 0$, and $C_{\kappa, 1} \in(0, \infty)$.

Proof. From (5.4) and that $G_{0} \equiv 0$, we get $C_{\kappa, t}=t C_{\kappa, 1}, t \geq 0$. From (5.3) and (5.4), we get $C_{\kappa, 1}>0$. Now we show $C_{\kappa, 1}<\infty$. Fix $z_{0}=x_{0}+i y_{0} \in \mathbb{H}$ and consider an $\operatorname{SLE}_{\kappa}(-8)$ process started from 0 with force point $z_{0}$. We will use some results in Appendix @ . We may express $T_{z_{0}}$ by (A.4), where $V_{s}$ is a diffusion process that satisfies the SDE (A.3) with initial value $V_{0}=\operatorname{arcsinh}\left(x_{0} / y_{0}\right)$. So we immediately have $T_{z_{0}} \geq y_{0}^{2} \int_{0}^{\infty} e^{-4 s} d s=y_{0}^{2} / 4$. Thus, $T_{z_{0}}>1$ if $y_{0}>2$. From (5.2) we see that $G_{1} \equiv 0$ on $\{\operatorname{Im} z>2\}$. From the left-right symmetry, we see that $G_{1}(x+i y)=G_{1}(-x+i y)$. We also know that $\left|G_{1}(z)\right| \leq|G(z)| \leq 1$ for all $z \in \mathbb{H}$. Thus, to show that $C_{\kappa, 1}=\int_{\mathbb{H}} G_{1}(z) d A(z)<\infty$, it suffices to prove that there is $M>0$ such that $\int_{M}^{\infty} \int_{0}^{2} G_{1}(x+i y) d y d x<\infty$.

Suppose $\left|\sqrt{\kappa} B_{s}\right| \leq 1+b s$ for every $s \geq 0$, where $b>0$ is to be determined. From A.3 and that $|\tanh (x)| \leq 1$, we have $V_{s} \geq V_{0}-1-b s-\left(\frac{\kappa}{2}+4\right) s, s \geq 0$. Thus,

$$
\cosh ^{2}\left(V_{2}\right) \geq \frac{1}{4} e^{2 V_{s}} \geq \frac{1}{4} e^{2 V_{0}} \cdot e^{-(2 b+\kappa+8) s-2} \geq e^{-(2 b+\kappa+8) s-2} \sinh ^{2}\left(V_{0}\right)=\frac{x_{0}^{2}}{y_{0}^{2}} e^{-(2 b+\kappa+8) s-2} .
$$

From (A.4) we then get $T_{z_{0}} \geq \frac{e^{-2} x_{0}^{2}}{\kappa+12+2 b}$, which then implies $T_{z_{0}}>1$ if $2 b<e^{-2} x_{0}^{2}-\kappa-12$. From A.5 know that $\mathbb{P}\left[\sqrt{\kappa}\left|B_{s}\right| \leq 1+b s, \forall s \geq 0\right] \geq 1-2 e^{-\frac{2}{\kappa} b}$. Thus, by taking $2 b=e^{-2} x_{0}^{2}-\kappa-12-\varepsilon$ and letting $\varepsilon \rightarrow 0^{+}$, we get

$$
\mathbb{P}_{z_{0}}^{\kappa,-8}\left[T_{z_{0}} \leq 1\right] \leq e^{-\frac{1}{\kappa}\left(e^{-2} x_{0}^{2}-\kappa-12\right)}, \quad \text { if } e^{-2} x_{0}^{2}>\kappa+12 .
$$

Let $M=\sqrt{e^{2}(\kappa+12)}$. From (5.2) we get

$$
G_{1}\left(z_{0}\right) \leq G\left(z_{0}\right) \mathbb{P}_{z_{0}}^{\kappa,-8}\left[T_{z_{0}} \leq 1\right] \leq \mathbb{P}_{z_{0}}^{\kappa,-8}\left[T_{z_{0}} \leq 1\right] \leq e^{-\frac{1}{\kappa}\left(e^{-2} x_{0}^{2}-\kappa-12\right)}, \quad x_{0}>M .
$$

So we get $\int_{M}^{\infty} \int_{0}^{2} G_{1}\left(z_{0}\right) d y d x<\infty$, as desired. 
Let $\mathrm{m}$ be the Lebesgue measure on $\mathbb{R}$. For any measurable set $U \subset \overline{\mathbb{H}}$, define

$$
\Theta_{t}^{\kappa,-8}(U)=C_{\kappa, 1} \mathrm{~m}\left(\gamma^{-1}(U) \cap[0, t]\right), \quad t \geq 0 .
$$

Especially, we have $\Theta_{t}^{\kappa,-8}:=\Theta_{t}^{\kappa,-8}(\overline{\mathbb{H}})=C_{\kappa, 1} t$. We can now state the following theorem, which is similar to Theorem 4.1 .

Theorem 5.1. For any measurable set $U \subset \overline{\mathbb{H}}$,

$$
\begin{gathered}
\mathbb{P}_{U}^{\kappa,-8} \widehat{\oplus} \mathbb{P}_{\kappa}=\mathbb{P}_{\kappa} \otimes d \Theta^{\kappa,-8}(U), \\
\mathcal{L}_{*}\left(\mathbb{P}_{z}^{\kappa,-8} \oplus \mathbb{P}_{\kappa}\right) \overleftarrow{\otimes} \mathbf{1}_{U} G^{\kappa,-8}(z) d A(z)=\mathcal{L}_{*}\left(\mathbb{P}_{\kappa}\right) \otimes \mathcal{M}_{U}^{\kappa,-8},
\end{gathered}
$$

where $\mathcal{M}_{U}^{\kappa,-8}$ is the kernel from $\mathcal{L}\left(\Sigma^{\mathcal{L}}\right)$ to $\overline{\mathbb{H}}$ defined by $\mathcal{M}_{U}^{\kappa,-8}(\gamma, \cdot)=\gamma_{*}\left(d \Theta^{\kappa,-8}(U)\right)$.

Proof. From the renewal property of Loewner's equation and the Markov property of $\sqrt{\kappa} B_{t}$, we see that, if $t \leq N$, then $M_{t}(z)-\mathbb{E}_{\kappa}\left[M_{N}(z) \mid \mathcal{F}_{t}^{0}\right]=\mathbf{1}_{T_{z}>t}\left|g_{t}^{\prime}(z)\right|^{2} G_{N-t}\left(g_{t}(z)-\lambda_{t}\right)$. Thus,

$$
\begin{gathered}
\int_{\mathbb{H}}\left(M_{t}(z)-\mathbb{E}_{\kappa}\left[M_{N}(z) \mid \mathcal{F}_{t}^{0}\right]\right) d A(z)=\int_{\mathbb{H} \backslash K_{t}} G_{N-t}\left(g_{t}(z)-\lambda_{t}\right)\left|g_{t}^{\prime}(z)\right|^{2} d A(z) \\
=\int_{\mathbb{H}} G_{N-t}(w) d A(w)=C_{\kappa, N-t}=(N-t) C_{\kappa, 1} .
\end{gathered}
$$

Here in the second "=" we use $w=g_{t}(z)-\lambda_{t}$ and the fact that $g_{t}$ maps $\mathbb{H} \backslash K_{t}$ conformally onto $\mathbb{H}$. The above formula together with Proposition 2.1 and (5.1) implies that $\int_{\mathbb{H}} \mathbb{P}_{z ; N}^{\kappa,-8} G(z) d A(z) \triangleleft \mathbb{P}_{\kappa}$, and the local Radon-Nikodym derivative is $C_{\kappa, 1}(N-t) \vee 0$. From Proposition 2.2 we get

$$
\int_{\mathbb{H}} \mathbb{P}_{z ; N}^{\kappa,-8} G(z) d A(z)=\mathcal{K}_{C_{\kappa, 1} d(t \wedge N)}\left(\mathbb{P}_{\kappa}\right) .
$$

Letting $N \rightarrow \infty$, we get

$$
\mathbb{P}_{\mathbb{H}}^{\kappa,-8}=\mathcal{K}_{C_{\kappa, 1} d t}\left(\mathbb{P}_{\kappa}\right)
$$

Using Proposition 2.4 we get

$$
\mathbb{P}_{\mathbb{H}}^{\kappa,-8} \widehat{\oplus} \mathbb{P}_{\kappa}=\left.\mathbb{P}_{\kappa} \otimes C_{\kappa, 1} \mathrm{~m}\right|_{[0, \infty)}
$$

Applying the extended Loewner map $\widehat{\mathcal{L}}(\lambda, t)=(\mathcal{L}(\lambda), \mathcal{L}(\lambda)(t))$, we get

$$
\mathcal{L}_{*}\left(\mathbb{P}_{z}^{\kappa,-8} \oplus \mathbb{P}_{\kappa}\right) \overleftarrow{\otimes} G(z) d A(z)=\mathcal{L}_{*}\left(\mathbb{P}_{\kappa}\right) \otimes \mathcal{M}^{\kappa,-8}
$$

where $\mathcal{M}^{\kappa,-8}$ is the kernel from $\mathcal{L}\left(\Sigma^{\mathcal{L}}\right)$ to $\overline{\mathbb{H}}$ defined by $\mathcal{M}_{U}^{\kappa,-8}(\gamma, \cdot)=\gamma_{*}\left(\left.C_{\kappa, 1} \mathrm{~m}\right|_{[0, \infty)}\right)$. Note that $\mathcal{M}_{U}^{\kappa,-8}(\gamma, \cdot)$ is the restriction of $\mathcal{M}^{\kappa,-8}(\gamma, \cdot)$ to $U$. So we get (5.6) by restricting both sides of (5.8) to $\Sigma^{\mathbb{C}} \times U$. Finally, we get (5.5) by applying $\widehat{\mathcal{L}}^{-1}$.

The following two corollaries are similar to Corollaries 4.1 and 4.2 .

Corollary 5.1. Let $U$ be a measurable subset of $\mathbb{H}$ with $\int_{U} G^{\kappa,-8}(z) d A(z)<\infty$. If we integrate the laws of extended $S L E_{\kappa}(-8)$ curve started from 0 with force point at $z$ against the measure $\mathbf{1}_{U} G^{\kappa,-8}(z) d A(z)$, then we get a finite measure on curves, which is absolutely continuous w.r.t. the law of an SLE $E_{\kappa}$ curve, and the Radon-Nikodym derivative is $\left|\mathcal{M}_{U}^{\kappa,-8}(\gamma, \cdot)\right|=C_{\kappa, 1} \mathrm{~m}\left(\gamma^{-1}(U)\right)$. 
Corollary 5.2. Suppose $(\gamma, t)$ is a $C([0, \infty), \mathbb{C}) \times[0, \infty)$-valued random variable with the properties that $\gamma$ has the law of an $S L E_{\kappa}$ curve, and given $\gamma$, the law of $t$ is absolutely continuous w.r.t. the Lebesgue measure on $[0, \infty)$. Then the law of $\gamma(t)$ is absolutely continuous w.r.t. $\mathbf{1}_{\mathbb{H}} d A(z)$, and the law of $\gamma$ conditioned on $z=\gamma(t)$ is absolutely continuous w.r.t. the law of an extended $S L E_{\kappa}(-8)$ curve started from 0 with force point at $z$.

Remark. From Corollary 5.2 we see that, if we sample a point on an $\mathrm{SLE}_{\kappa}$ curve according to a law that is absolutely continuous w.r.t. the capacity parametrization, and stop the $\mathrm{SLE}_{\kappa}$ curve at that point, then the law of the stopped curve conditioned on that point is absolutely continuous w.r.t. the law of $\operatorname{SLE}_{\kappa}(-8)$ curve. This extends a result in [32, whose argument used the symmetry of backward $\mathrm{SLE}_{\kappa}$ welding for $\kappa \in(0,4]$ (cf. [25]) and the conformal removability of $\mathrm{SLE}_{\kappa}$ curves for $\kappa \in(0,4)$ (cf. [10, 24]).

More specifically, from [32, Remark 2 after Theorem 6.6], we know that, if $\kappa \in(0,4)$, then the above conditional stopped curve is the conformal image of an initial segment of a whole-plane $\operatorname{SLE}_{\kappa}(\kappa+2)$ curve, which is also an end segment of a whole-plane $\operatorname{SLE}_{\kappa}(\kappa+2)$ curve, thanks to the reversibility of whole-plane $\operatorname{SLE}_{\kappa}(\rho)$ curve (cf. [22]). From Proposition ??, we know that an end segment of a whole-plane $\operatorname{SLE}_{\kappa}(\kappa+2)$ curve can be mapped conformally to an end segment of a chordal $\operatorname{SLE}_{\kappa}(-8)$ curve. Thus, Corollary 5.2 extends a weaker version of [32, Remark 2 after Theorem 6.6] from $\kappa \in(0,4)$ to $\kappa \in(0, \infty)$. The result here is weaker because we can not conclude that the conditional stopped curve is exactly the conformal image of an $\operatorname{SLE}_{\kappa}(-8)$ curve, but can only say that its law is absolutely continuous w.r.t. the law of an $\operatorname{SLE}_{\kappa}(-8)$ curve.

Corollary 5.3. Let $\gamma$ be an $S L E_{\kappa}$ curve. Then for any measurable set $U \subset \overline{\mathbb{H}}$, we have $\mathbb{E}\left[\mathrm{m}\left(\gamma^{-1}(U)\right)\right]=\frac{1}{C_{\kappa, 1}} \int_{U} G^{\kappa,-8}(z) d A(z)$. Especially, we have a.s. $\mathrm{m}\left(\gamma^{-1}(\mathbb{R})\right)=0$.

Proof. The first statement follows from computing the total mass of the measures in (5.6) and the fact that $\left|\mathcal{M}_{U}^{\kappa,-8}\right|=C_{\kappa, 1} \mathrm{~m}\left(\gamma^{-1}(U)\right)$. The second statement follows from taking $U=\mathbb{R}$ and the fact that $\int_{R} G^{\kappa,-8}(z) d A(z)=0$.

Remark. The above corollary says that $\frac{1}{C_{\kappa, 1}} G^{\kappa,-8}(z)$ is the density of $\mathrm{SLE}_{\kappa}$ curve in capacity parametrization, and so may be called the capacity Green's function for $\mathrm{SLE}_{\kappa}$.

Corollary 5.4. Let $\gamma$ be an $S L E_{\kappa}$ curve. Then there is a random conformal map $W$ from $\mathbb{H}$ into $\widehat{\mathbb{C}}$ such that $W(\gamma(1))=\infty$, and the law of $W(\gamma(t)), 0 \leq t \leq 1$, is absolutely continuous w.r.t. an end segment of a whole-plane $S L E_{\kappa}(\kappa+2)$ curve, up to a reparametrization.

Proof. Let $\widetilde{\gamma}$ be an $\operatorname{SLE}_{\kappa}$ curve independent of $\gamma$. Let $\widetilde{\lambda}_{t}$ and $\widetilde{g}_{t}$ be the driving function and Loewner maps associated with $\widetilde{\gamma}$. Let $\xi$ be a random variable with law $\mathbf{1}_{[0,1]} d x$ that is independent of $\widetilde{\gamma}$ and $\gamma$. Define $\widehat{\gamma}$ such that $\widehat{\gamma}(t)=\widetilde{\gamma}(t)$ for $0 \leq t \leq \xi$ and $\widehat{\gamma}(t)=$ $\widetilde{f}_{\xi}\left(\widetilde{\lambda}_{\xi}+\gamma(t-\xi)\right)$ for $\xi \leq t<\infty$, where $\widetilde{f}_{\xi}$ is the continuation of $\widetilde{g}_{\xi}^{-1}$ to $\overline{\mathbb{H}}$. From the domain Markov property of $\mathrm{SLE}_{\kappa}$, we see that $\widehat{\gamma}$ is an $\mathrm{SLE}_{\kappa}$ curve independent of $\xi$. Since $\xi+1$ has the law $\mathbf{1}_{[1,2]} d x$, from Corollary [5.2, we see that, conditioned on $z_{0}=\widehat{\gamma}(\xi+1)$, the law of $\widehat{\gamma}_{t}, 0 \leq t \leq \xi+1$, is absolutely continuous w.r.t. the law of an $\operatorname{SLE}_{\kappa}(-8)$ curve started from 0 with force point at $z_{0}$. Let $W_{1}$ be a conformal map from $\mathbb{H}$ onto $\mathbb{D}:=\{|z|<1\}$ such that $W_{1}(0)=1$ and $W_{1}\left(z_{0}\right)=0$. From Proposition ??, we know that $W_{1}$ maps an $\operatorname{SLE}_{\kappa}(-8)$ curve started from 0 with force point at $z_{0}$ to a radial $\operatorname{SLE}_{\kappa}(\kappa+2)$ curve in $\mathbb{D}$ started from 1 with force point at $W_{1}(\infty)$, up to a reparametrization. 
By [1], there is a conformal map $W_{2}$ from $\mathbb{D}$ into $\widehat{\mathbb{C}}$ such that $W_{2}(0)=\infty$, and $W_{2}$ maps the radial $\mathrm{SLE}_{\kappa}(\kappa+2)$ curve to an end segment of a whole-plane $\mathrm{SLE}_{\kappa}(\kappa+2)$ curve. Let $W_{0}(z)=\widetilde{f}_{\xi}\left(\widetilde{\lambda}_{\xi}+z\right)$, which is a conformal map from $\mathbb{H}$ into $\mathbb{H}$. Then $W:=W_{2} \circ W_{1} \circ W_{0}$ is the conformal map we are looking for.

Remark. Corollary [5.4 extends a weaker version of [32, Theorem 5.3], which states that, for $\kappa \in(0,4)$, there is a random conformal map $W$ from $\mathbb{H}$ into $\widehat{\mathbb{C}}$ such that $W(\gamma(1))=0$, and $W(\gamma(t)), 0 \leq t \leq 1$, is an initial segment of a whole-plane $\operatorname{SLE}_{\kappa}(\kappa+2)$ curve, up to a reparametrization.

Corollary 5.5. If $\int_{U} G^{\kappa,-8}(z) d A(z)<\infty$, then $M_{t}^{\kappa,-8}(U):=\Psi_{t}^{\kappa,-8}(U)+\Theta_{t}^{\kappa,-8}(U)$ is a uniformly integrable $\left(\mathcal{F}_{t}^{0}\right)$-martingale.

Proof. It suffices to show that $M_{t}^{\kappa,-8}(U)=\mathbb{E}_{\kappa}\left[C_{\kappa, 1} \mathrm{~m}\left(\gamma^{-1}(U)\right) \mid \mathcal{F}_{t}^{0}\right]$ for every $t \geq 0$. We have $C_{\kappa, 1} \mathrm{~m}\left(\gamma^{-1}(U)\right)=C_{\kappa, 1} \mathrm{~m}\left(\gamma^{-1}(U) \cap[0, t]\right)+C_{\kappa, 1} \mathrm{~m}\left(\gamma^{-1}(U) \cap[t, \infty]\right)$, and $C_{\kappa, 1} \mathrm{~m}\left(\gamma^{-1}(U) \cap\right.$ $[0, t])=\Theta_{t}^{\kappa,-8}(U)$ is $\mathcal{F}_{t}^{0}$-measurable. Thus, it remains to show that

$$
\mathbb{E}_{\kappa}\left[C_{\kappa, 1} \mathrm{~m}\left(\gamma^{-1}(U) \cap\left[t_{0}, \infty\right]\right) \mid \mathcal{F}_{t_{0}}^{0}\right]=\Psi_{t_{0}}^{\kappa,-8}(U), \quad t_{0} \geq 0 .
$$

Fix $t_{0} \geq 0$. By the domain Markov Property of $\mathrm{SLE}_{\kappa}$, there is an $\mathrm{SLE}_{\kappa}$ curve $\widehat{\gamma}$ independent of $\mathcal{F}_{t_{0}}^{0}$ such that $\gamma\left(t_{0}+t\right)=f_{t_{0}}\left(\lambda_{t_{0}}+\widehat{\gamma}(t)\right)$ for all $t \geq 0$, where $f_{t_{0}}$ is the continuation of $g_{t_{0}}^{-1}$ to $\overline{\mathbb{H}}$, and $\lambda_{t}$ and $g_{t}$ are the driving function and Loewner maps associated with $\gamma$. Let $\widetilde{g}_{t_{0}}(z)=g_{t_{0}}(z)-\lambda_{t_{0}}$ and $\widetilde{f}_{t_{0}}(z)=f_{t_{0}}\left(\lambda_{t_{0}}+z\right)$. Conditioned on $\mathcal{F}_{t_{0}}^{0}$, we have

$$
\begin{gathered}
\mathrm{m}\left(\gamma^{-1}(U) \cap\left[t_{0}, \infty\right]\right)=\mathrm{m}\left(\widehat{\gamma}^{-1}\left(\widetilde{f}_{t_{0}}^{-1}(U)\right)\right)=\mathrm{m}\left(\widehat{\gamma}^{-1}\left(\widetilde{f}_{t_{0}}^{-1}(U) \cap \mathbb{H}\right)\right) \\
=\mathrm{m}\left(\widehat{\gamma}^{-1}\left(\widetilde{g}_{t_{0}}\left(U \cap\left(\mathbb{H} \backslash K_{t_{0}}\right)\right)\right)\right.
\end{gathered}
$$

From Corollary 5.3, we get

$$
\begin{gathered}
\mathbb{E}_{\kappa}\left[\mathrm{m}\left(\gamma^{-1}(U) \cap\left[t_{0}, \infty\right]\right) \mid \mathcal{F}_{t_{0}}^{0}\right]=\int_{\widetilde{g}_{t_{0}}\left(U \cap\left(\mathbb{H} \backslash K_{t_{0}}\right)\right)} G^{\kappa,-8}(z) d A(z) \\
=\int_{U \cap\left(\mathbb{H} \backslash K_{t_{0}}\right)} G^{\kappa,-8}\left(g_{t_{0}}(z)-\lambda_{t_{0}}\right)\left|g_{t_{0}}^{\prime}(z)\right|^{2} d A(z)=\int_{U} M_{t_{0}}^{\kappa,-8}(z) d A(z)=\Psi_{t_{0}}^{\kappa,-8}(U) .
\end{gathered}
$$

This finishes the proof.

\section{Intersection of SLE with the boundary}

In this section, we decompose $\operatorname{SLE}_{\kappa}$ into $\operatorname{SLE}_{\kappa}(\rho)$ processes with the force point lying on $\mathbb{R}$. An $\operatorname{SLE}_{\kappa}(\rho)$ process started from $a_{0} \in \mathbb{R}$ with force point at $x_{0} \in \mathbb{R} \backslash\left\{a_{0}\right\}$ is the solution of the Loewner equation driven by $\lambda_{t}, 0 \leq t<T_{x_{0}}$, which is the solution of the SDE:

$$
d \lambda_{t}=\sqrt{\kappa} d B_{t}+\frac{\rho}{\lambda_{t}-g_{t}^{\lambda}\left(x_{0}\right)}, \quad \lambda_{0}=a_{0}
$$

The Loewner curve driven by $\lambda$, which a.s. exists, is called an $\operatorname{SLE}_{\kappa}(\rho)$ curve started from $a_{0}$ with force point at $x_{0}$. 
Let $\mathbb{P}_{x_{0}, \rho}$ denote the law of the driving $\operatorname{SLE}_{\kappa}(\rho)$ process started from 0 with force point at $x_{0} \in \mathbb{R} \backslash\{0\}$. From Proposition 2.3. we know that $\mathbb{P}_{x_{0}}^{\kappa, \rho} \triangleleft \mathbb{P}_{\kappa}$. We now derive the local Radon-Nikodym derivative. Let $\lambda_{t}=\sqrt{\kappa} B_{t}$ and $g_{t}$ be the Loewner maps driven by $\lambda$. Define

$$
M_{t}^{\kappa, \rho}\left(x_{0}\right)=\left|g_{t}\left(x_{0}\right)-\lambda_{t}\right|^{\frac{\rho}{\kappa}} \cdot g_{t}^{\prime}\left(x_{0}\right)^{\frac{\rho}{\kappa}\left(1-\frac{\kappa}{4}+\frac{\rho}{4}\right)}, \quad 0 \leq t<T_{x_{0}},
$$

and

$$
G^{\kappa, \rho}\left(x_{0}\right)=M_{0}^{\kappa, \rho}\left(x_{0}\right)=\left|x_{0}\right|^{\frac{\rho}{\kappa}}, \quad z \in \mathbb{H} .
$$

Direct calculation using Itô's formula shows that $\left(M_{t}^{\kappa, \rho}\left(x_{0}\right)\right)$ is an $\left(\mathcal{F}_{t}^{0}\right)$-adapted continuous local martingale, and satisfies the SDE:

$$
d M_{t}^{\kappa, \rho}\left(x_{0}\right)=M_{t}^{\kappa, \rho}\left(x_{0}\right) \cdot \frac{\rho / \sqrt{\kappa}}{\lambda_{t}-g_{t}\left(x_{0}\right)} d B_{t}, \quad 0 \leq t<T_{x_{0}} .
$$

We further define $M_{t}^{\kappa, \rho}\left(x_{0}\right)=0$ for $t \in\left[T_{x_{0}}, \infty\right)$. From Proposition 2.3 we know that

$$
\frac{\left.d \mathbb{P}_{x_{0}}^{\kappa, \rho}\right|_{\mathcal{F}_{t}^{0} \cap \Sigma_{t}}}{\left.d \mathbb{P}_{\kappa}\right|_{\mathcal{F}_{t}^{0} \cap \Sigma_{t}}}=\frac{M_{t}^{\kappa, \rho}\left(x_{0}\right)}{G^{\kappa, \rho}\left(x_{0}\right)}, \quad 0 \leq t<\infty .
$$

For a measurable subset $U$ of $\mathbb{R}$, define

$$
\Psi_{t}^{\kappa, \rho}(U)=\int_{U} M_{t}^{\kappa, \rho}(x) d x, \quad \mathbb{P}_{U}^{\kappa, \rho}=\int_{U} \mathbb{P}_{x}^{\kappa, \rho} G^{\kappa, \rho}(x) d x
$$

For each $x \in \mathbb{R} \backslash\{0\},\left(M_{t}^{\kappa, \rho}(x)\right)$ is a supermartingale because it is a positive local martingale. Thus, if $\int_{U} G^{\kappa, \rho}(x) d x<\infty$, then $\left(\Psi_{t}^{\kappa, \rho}(U)\right)$ is also a supermartingale, which has to be $\mathbb{P}_{\kappa}$-a.s. finite. From Proposition 2.1, we have

$$
\frac{\left.d \mathbb{P}_{U}^{\kappa, \rho}\right|_{\mathcal{F}_{t}^{0} \cap \Sigma_{t}}}{\left.d \mathbb{P}_{\kappa}\right|_{\mathcal{F}_{t}^{0} \cap \Sigma_{t}}}=\Psi_{t}^{\kappa, \rho}(U), \quad 0 \leq t<\infty .
$$

It is known that (cf. [30]), if $\rho \leq \frac{\kappa}{2}-4$, then for an $\operatorname{SLE}_{\kappa}(\rho)$ curve $\gamma$ with force point $x_{0}$, we have a.s. $\lim _{t \rightarrow T_{x_{0}}^{-}} \gamma(t)=x_{0}$, which then implies that $T_{x_{0}}<\infty$, and $\lim _{t \rightarrow T_{x_{0}}^{-}} \lambda_{t}$ converges, where $\lambda$ is the corresponding driving function. This means that $\mathbb{P}_{x_{0}}^{\kappa, \rho}$ is supported by $\Sigma^{\oplus}$, and we may define $\mathbb{P}_{x_{0}}^{\kappa, \rho} \oplus \mathbb{P}_{\kappa}$. The pushforward measure $\mathcal{L}_{*}\left(\mathbb{P}_{x_{0}}^{\kappa, \rho} \oplus \mathbb{P}_{\kappa}\right)$ is called the law of an extended $\operatorname{SLE}_{\kappa}(\rho)$ curve started from 0 with force point at $x_{0}$.

For $\kappa \in(0,8)$ and $\rho=\kappa-8$, an extended $\operatorname{SLE}_{\kappa}(\rho)$ curve with force point at $x_{0}$ is also called a two-sided chordal $\mathrm{SLE}_{\kappa}$ curve through $x_{0}$. It can be understood as an $\mathrm{SLE}_{\kappa}$ curve conditioned to pass though $x_{0}$. To make this rigorous, one may condition an SLE $_{\kappa}$ curve $\gamma$ on the event that $\operatorname{dist}\left(x_{0}, \gamma\right)<r$, and then pass the limit $r \rightarrow 0$. A two-sided chordal SLE $\mathrm{S}_{\kappa}$ curve also satisfies reversibility, which is similar to that of a two-sided radial $\mathrm{SLE}_{\kappa}$ curve.

It is proved in [2] that, for $\kappa \in(4,8)$, if $U$ is a pre-compact measurable subset of $\mathbb{R} \backslash\{0\}$, then $\left(\Psi_{t}^{\kappa, \kappa-8}(U)\right)$ is of class $\mathcal{D}$, and Doob-Meyer decomposition theorem can be applied to get a unique continuous increasing function $\Theta_{t}(U)$ such that $\Theta_{0}(U)=0$ and $\Psi_{t}(U)+\Theta_{t}(U)$ is a uniformly integrable martingale. We may then define $\Theta_{t}(U)$ for any measurable subset $U$ of $\mathbb{R}$ using a limiting procedure. It is conjectured (cf. [2] and [13]) that $\Theta_{t}(U)$ agrees up to a multiplicative constant with the $d^{\prime}$-dimensional Minkowski content of $\gamma([0, t]) \cap U$, where $d^{\prime}:=2-\frac{8}{\kappa}$ is the Hausdorff dimension of $\gamma \cap \mathbb{R}$ (cf. 3]). Using the argument in Section 4 . we can obtain the following theorem and corollaries. 
Theorem 6.1. Let $\kappa \in(4,8)$. Let $U$ be any measurable subset of $\mathbb{R}$. Then we have

$$
\begin{gathered}
\mathbb{P}_{U}^{\kappa, \kappa-8} \widehat{\oplus} \mathbb{P}_{\kappa}=\mathbb{P}_{\kappa} \otimes d \Theta(U), \\
\mathcal{L}_{*}\left(\mathbb{P}_{x}^{\kappa, \kappa-8} \oplus \mathbb{P}_{\kappa}\right) \overleftarrow{\otimes} \mathbf{1}_{U} G^{\kappa, \kappa-8}(x) d x=\mathcal{L}_{*}\left(\mathbb{P}_{\kappa}\right) \otimes \mathcal{M}_{U},
\end{gathered}
$$

where $\mathcal{M}_{U}$ is the $\mathcal{L}_{*}\left(\mathbb{P}_{\kappa}\right)$-kernel from $\mathcal{L}\left(\Sigma^{\mathcal{L}}\right)$ to $\overline{\mathbb{H}}$ defined by $\mathcal{M}_{U}(\gamma, \cdot)=\gamma_{*}(d \Theta(U))$.

Corollary 6.1. Let $\kappa \in(4,8)$. Let $U$ be a measurable subset of $\mathbb{R}$ with $\int_{U} G^{\kappa, \kappa-8}(x) d x<$ $\infty$. If we integrate the laws of two-sided chordal $S L E_{\kappa}$ through $x$ against the measure $\mathbf{1}_{U} G^{\kappa, \kappa-8}(x) d x$, then we get a finite measure on curves, which is absolutely continuous w.r.t. the law of an $S L E_{\kappa}$ curve, and the Radon-Nikodym derivative is $\left|\mathcal{M}_{U}(\gamma, \cdot)\right|=\Theta_{\infty}(U)\left(\mathcal{L}^{-1}(\gamma)\right)$.

Corollary 6.2. Let $\kappa \in(4,8)$. Suppose $(\gamma, x)$ is a $C([0, \infty), \mathbb{C}) \times \mathbb{R}$-valued random variable with the properties that $\gamma$ has the law of an $S L E_{\kappa}$ curve, and given $\gamma$, the law of $x$ is absolutely continuous w.r.t. $\mathcal{M}_{\mathbb{R}}(\gamma, \cdot)$. Then the law of $x$ is absolutely continuous w.r.t. $\mathrm{m}$, and the law of $\gamma$ given $x$ is absolutely continuous w.r.t. the law of a two-sided chordal SLE $E_{\kappa}$ curve through $x$.

Remark. Since an $\mathrm{SLE}_{\kappa}$ curve for $\kappa \in(0,4]$ does not visit any point on $\mathbb{R} \backslash\{0\}$, Corollary 6.1 does not hold in the case $\kappa \in(0,4]$, and neither does the main result in [2].

\section{Decomposition of Planar Brownian motion}

We now use the argument in the proof of Theorem 4.1 to decompose a planar Brownian motion. We modify the definition of $\Sigma$ such that the continuous functions take values in $\mathbb{R}^{2}$. Let $D \subset \mathbb{R}^{2}$ be a bounded domain that contains $0=(0,0)$. Let $B_{t}$ be a planar Brownian motion that starts from 0 , and $\tau_{D}$ the first time that $B_{t}$ exists $D$. Let $G_{D}(\cdot, \cdot)$ denote the Dirichlet Green's function in $D$ for Brownian motion. Fix $z_{0}=\left(x_{0}, y_{0}\right) \in D \backslash\{0\}$. Consider the Doob's $h$-transform of $B_{t}$ with $h=G_{D}\left(\cdot, z_{0}\right)$. This a diffusion process that satisfies the SDE:

$$
d Z_{t}^{z_{0}}=d B_{t}+\frac{\left.\nabla G_{D}\left(\cdot, z_{0}\right)\right|_{Z_{t}^{z_{0}}}}{G_{D}\left(Z_{t}^{z_{0}}, z_{0}\right)} d t, \quad Z_{0}^{z_{0}}=0 .
$$

We may view $Z_{t}^{z_{0}}$ as $B_{t}$ conditioned to visit $z_{0}$ before exiting $D$. Let $\mathbb{P}$ and $\mathbb{P}_{z_{0}}^{D}$ denote the laws of $\left(B_{t}\right)$ and $\left(Z_{t}^{z_{0}}\right)$, respectively. Then $\mathbb{P}_{z_{0}}^{D} \triangleleft \mathbb{P}$, and

$$
\frac{\left.d \mathbb{P}_{z_{0}}^{D}\right|_{\mathcal{F}_{t}^{0} \cap \Sigma_{t}}}{\left.d \mathbb{P}\right|_{\mathcal{F}_{t}^{0} \cap \Sigma_{t}}}=\frac{G_{D}\left(B_{t \wedge \tau_{D}}, z_{0}\right)}{G_{D}\left(0, z_{0}\right)}, \quad 0 \leq t<\infty
$$

Let $f(w)=\int_{D} G_{D}(w, z) d A(z)$. Then $f$ is the solution of the Dirichlet problem $\Delta f \equiv-1$ in $D$ and $f \equiv 0$ on $\partial D$. Define

$$
\mathbb{P}^{D}=\int_{D} \mathbb{P}_{z}^{D} G_{D}(0, z) d A(z), \quad \Psi_{t}=\int_{D} G_{D}\left(B_{t \wedge \tau_{D}}, z\right) d A(z)=f\left(B_{t \wedge \tau_{D}}\right) .
$$

From Proposition 2.1, we see that $\mathbb{P}^{D} \triangleleft \mathbb{P}$ and

$$
\frac{\left.d \mathbb{P}^{D}\right|_{\mathcal{F}_{t}^{0} \cap \Sigma_{t}}}{\left.d \mathbb{P}\right|_{\mathcal{F}_{t}^{0} \cap \Sigma_{t}}}=\Psi_{t}, \quad 0 \leq t<\infty .
$$


From Itô's formula and the PDE for $f$, we see that $M_{t}:=\Psi_{t}+\frac{t}{2}, 0 \leq t<\tau_{D}$, is a continuous local martingale, and $\lim _{t \rightarrow \tau_{D}^{-}} M_{t}=\frac{1}{2} \tau_{D}$. We further define $M_{t}=\frac{1}{2} \tau_{D}$ for $t \geq \tau_{D}$. Since $D$ is bounded, the probability $\mathbb{P}\left[\tau_{D}>N\right]$ decays exponentially as $N \rightarrow \infty$. Also note that $f$ is bounded. Thus, $\left(M_{t}\right)$ is a uniformly integrable martingale, and we have

$$
\mathbb{E}\left[\frac{\tau_{D}}{2} \mid \mathcal{F}_{t}^{0}\right]=\mathbb{E}\left[M_{\infty} \mid \mathcal{F}_{t}^{0}\right]=M_{t}=\Psi_{t}+\frac{t \wedge \tau_{D}}{2}, \quad 0 \leq t<\infty .
$$

Define a process $\left(\theta_{t}\right)$ such that $\theta_{t}=\frac{t \wedge \tau_{D}}{2}$. Then we have

$$
\mathbb{E}\left[\theta_{\infty} \mid \mathcal{F}_{t}^{0}\right]-\theta_{t}=\Psi_{t}, \quad 0 \leq t<\infty .
$$

From Proposition 2.2, (7.1) and (7.2), we see that

$$
\mathbb{P}^{D}=\mathcal{K}_{d \theta}(\mathbb{P}) .
$$

Apply first the operation $\widehat{\oplus} \mathbb{P}$ and then the pushforward by the map $(B, t) \mapsto(B, B(t))$ to both sides of (7.3). Using a variation of Proposition 2.4, we obtain the following theorem.

Theorem 7.1. Let $\mathrm{m}$ and $d A$ denote the Lebesgue measures on $\mathbb{R}$ and $\mathbb{R}^{2}$, respectively. Then we have

$$
\left(\mathbb{P}_{z}^{D} \oplus \mathbb{P}\right) \overleftarrow{\otimes} G_{D}(z, 0) d A(z)=\mathbb{P} \otimes \mathcal{M}
$$

where $\mathcal{M}$ is a kernel from $\Sigma$ to $D$ defined by $\mathcal{M}(B, \cdot)=\frac{1}{2} B_{*}\left(\left.\mathrm{~m}\right|_{\left[0, \tau_{D}\right]}\right)$.

\section{Appendices}

\section{A Boundedness of $\operatorname{SLE}_{\kappa}(\rho)$}

In this section, we prove Proposition 3.1(i). Fix $\kappa>0$ and $\rho \leq \frac{\kappa}{2}-4$. Fix $z_{0}=x_{0}+i y_{0} \in \mathbb{H}$. Let $\gamma$ be an $\operatorname{SLE}_{\kappa}(\rho)$ curve with force point at $z_{0}$. Let $\lambda(t), 0 \leq t<T_{z_{0}}$, be the driving function, and $g_{t}$ be the corresponding Loewner maps. Define $X_{t}, Y_{t}, D_{t}$ using (3.1). Then the ODEs (3.2) still hold, and now $X_{t}$ satisfies the SDE:

$$
d X_{t}=-\sqrt{\kappa} d B_{t}+\frac{(\rho+2) X_{t}}{X_{t}^{2}+Y_{t}^{2}} d t
$$

Let $u(t)=\int_{0}^{t} \frac{1}{X_{s}^{2}+Y_{s}^{2}} d s$. Let $\widehat{X}_{s}=X_{u^{-1}(s)}, \widehat{Y}_{s}=Y_{u^{-1}(s)}$, and $\widehat{R}_{s}=\frac{\widehat{X}_{s}}{\widehat{Y}_{s}}$. Using (3.2) and (A.1), we find that $\widehat{Y}_{s}=y_{0} e^{-2 s}$ and there is a standard Brownian motion $\widehat{B}_{s}$ such that

$$
d \widehat{R}_{s}=\sqrt{\kappa} \sqrt{1+\widehat{R}_{s}^{2}} d \widehat{B}_{s}+(\rho+4) \widehat{R}_{s} d s .
$$

Let $V_{s}=\operatorname{arcsinh}\left(\widehat{R}_{s}\right)$. From Itô's formula, we get

$$
d V_{s}=\sqrt{\kappa} d \widehat{B}_{s}+\left(\rho+4-\frac{\kappa}{2}\right) \tanh \left(V_{s}\right) d s .
$$

From $u(t)=\int_{0}^{t} \frac{1}{X_{s}^{2}+Y_{s}^{2}} d s, \widehat{Y}_{s}=y_{0} e^{-2 s}$, and $V_{s}=\operatorname{arcsinh}\left(\widehat{R}_{s}\right)$, we get

$$
T_{z_{0}}=\int_{0}^{\infty} \widehat{Y}_{s}^{2}\left(1+\widehat{R}_{s}^{2}\right) d s=y_{0}^{2} \int_{0}^{\infty} e^{-4 s} \cosh ^{2}\left(V_{s}\right) d s .
$$


Lemma A.1. Suppose $\left(V_{s}\right)$ solves (A.3) with $\rho \leq \frac{\kappa}{2}-4$. Then $\left(V_{s}\right)$ can be coupled with a standard Brownian motion $\widetilde{B}_{s}$ such that $\left|V_{s}\right| \leq\left|V_{0}+\sqrt{\kappa} \widetilde{B}_{s}\right|$ for all $s$.

Proof. From Itô-Tanaka's formula, $\left|V_{s}\right|$ satisfies the SDE:

$$
d\left|V_{s}\right|=\operatorname{sign}\left(V_{s}\right) \sqrt{\kappa} d \widehat{B}_{s}+\left(\rho+4-\frac{\kappa}{2}\right) \tanh \left(\left|V_{s}\right|\right) d s+d L_{s}^{V},
$$

where $L^{V}$ is continuous and increasing, and stays constant on the intervals on which $\mid V$. $\mid>0$. On the other hand, if $\widetilde{B}_{s}$ is a standard Brownian motion, then $\left|V_{0}+\sqrt{\kappa} \widetilde{B}_{s}\right|$ satisfies the SDE:

$$
d\left|V_{0}+\sqrt{\kappa} \widetilde{B}_{s}\right|=\operatorname{sign}\left(V_{0}+\sqrt{\kappa} \widetilde{B}_{s}\right) \sqrt{\kappa} d \widetilde{B}_{s}+d L_{s}^{B},
$$

where $L^{B}$ is continuous and increasing. Define two standard Brownian motions $B_{t}^{(1)}$ and $B_{t}^{(2)}$ such that $B_{t}^{(1)}=\int_{0}^{t} \operatorname{sign}\left(V_{s}\right) d \widehat{B}_{s}$ and $B_{t}^{(2)}=\int_{0}^{t} \operatorname{sign}\left(V_{0}+\sqrt{\kappa} \widetilde{B}_{s}\right) d \widetilde{B}_{s}$. We may couple $\widetilde{B}_{s}$ with $\widehat{B}_{s}$, and so with $V_{s}$, such that $B^{(1)}=B^{(2)}$. Since $\rho+4-\frac{\kappa}{2} \leq 0$, from the SDE for $\left|V_{s}\right|$ and $\left|V_{0}+\sqrt{\kappa} \widetilde{B}_{s}\right|$, we find that $\left|V_{s}\right|-\sqrt{\kappa} B_{s}^{(1)}-L_{s}^{V}$ is decreasing, and $\left|V_{0}+\sqrt{\kappa} \widetilde{B}_{s}\right|-$ $\sqrt{\kappa} B_{s}^{(1)}$ is increasing. Suppose that there is $s_{0} \geq 0$ such that $\left|V_{s_{0}}\right|>\left|V_{0}+\sqrt{\kappa} \widetilde{B}_{s_{0}}\right|$. Then $s_{0}>0$. Let $s_{0}^{\prime} \in\left(0, s_{0}\right)$ be such that $\left|V_{s_{0}^{\prime}}\right|=\left|V_{0}+\sqrt{\kappa} \widetilde{B}_{s_{0}^{\prime}}\right|$ and $\left|V_{s}\right|>\left|V_{0}+\sqrt{\kappa} \widetilde{B}_{s}\right|$ for $s \in\left(s_{0}^{\prime}, s_{0}\right]$. Then $\left|V_{s}\right|>0$ on $\left(s_{0}^{\prime}, s_{0}\right]$. So $L_{s}^{V}$ stays constant on $\left[s_{0}^{\prime}, s_{0}\right]$, which implies that $\left|V_{s}\right|-\sqrt{\kappa} B_{s}^{(1)}$ is decreasing on $\left[s_{0}^{\prime}, s_{0}\right]$. Since $\left|V_{0}+\sqrt{\kappa} \widetilde{B}_{s}\right|-\sqrt{\kappa} B_{s}^{(1)}$ is increasing, we conclude that $\left|V_{s}\right|-\left|V_{0}+\sqrt{\kappa} \widetilde{B}_{s}\right|$ is decreasing on $\left[s_{0}^{\prime}, s_{0}\right]$, which contradicts that $\left|V_{s_{0}}\right|>\left|V_{0}+\sqrt{\kappa} \widetilde{B}_{s_{0}}\right|$ and $\left|V_{s_{0}^{\prime}}\right|=\left|V_{0}+\sqrt{\kappa} \widetilde{B}_{s_{0}^{\prime}}\right|$. Thus, $\left|V_{s}\right| \leq\left|V_{0}+\sqrt{\kappa} \widetilde{B}_{s}\right|$ for all $s \geq 0$.

We will use the following well-known inequalities about Brownian motions:

$$
\mathbb{P}\left[\left|\sqrt{\kappa} B_{t}\right| \leq a t+b, \forall t \in[0, \infty)\right] \geq 1-2 e^{-\frac{2 a b}{\kappa}}, \quad a, b>0 .
$$

Proof of Proposition 3.1 (i). From (A.4) we get $T_{z_{0}} \leq y_{0}^{2} \int_{0}^{\infty} e^{-4 s} e^{2\left|V_{s}\right|} d s$. From Lemma A.1 $\left(V_{s}\right)$ may be coupled with a standard Brownian motion $\widetilde{B}_{s}$ such that $\left|V_{s}\right| \leq\left|V_{0}+\sqrt{\kappa} \widetilde{B}_{s}\right|$. Since $e^{2\left|V_{0}\right|} \leq 4 \cosh ^{2}\left(V_{0}\right)=4\left(1+\widehat{R}_{0}^{2}\right)=4 \frac{\left|z_{0}\right|^{2}}{y_{0}^{2}}$, we get

$$
T_{z_{0}} \leq y_{0}^{2} \int_{0}^{\infty} e^{-4 s} e^{2\left|V_{0}\right|+2 \sqrt{\kappa}\left|\widetilde{B}_{s}\right|} d s \leq 4\left|z_{0}\right|^{2} \int_{0}^{\infty} e^{2 \sqrt{\kappa}\left|\widetilde{B}_{s}\right|-4 s} d s .
$$

From (A.5) we see that the probability that $\sqrt{\kappa}\left|\widetilde{B}_{s}\right| \leq s+b$ for all $s \geq 0$ is at least $1-2 e^{-\frac{2}{\kappa} b}$, and on this event, from the above formula we get $T_{z_{0}} \leq 2\left|z_{0}\right|^{2} e^{2 b}$. Thus,

$$
\mathbb{P}_{z_{0}}^{\kappa, \rho}\left[T_{z_{0}} \leq 2\left|z_{0}\right|^{2} e^{2 b}\right] \geq 1-2 e^{-\frac{2}{\kappa} b}, \quad b>0 .
$$

This implies that $\mathbb{P}_{z_{0}}^{\kappa, \rho}$-a.s., $T_{z_{0}}<\infty$.

Recall that $\lambda_{t}=\sqrt{\kappa} B_{t}+\int_{0}^{t} \frac{-\rho X_{r}}{X_{r}^{2}+Y_{r}^{2}} d r, 0 \leq t<T_{z_{0}}$. The finiteness of $T_{z_{0}}$ implies that a.s. $\lim _{t \rightarrow T_{z_{0}}^{-}} B_{t} \in \mathbb{R}$. For the other term, consider

$$
\int_{0}^{T_{z_{0}}} \frac{\left|X_{t}\right|}{X_{t}^{2}+Y_{t}^{2}} d t=\int_{0}^{\infty}\left|\widehat{X}_{s}\right| d s=\int_{0}^{\infty} \widehat{Y}_{s}\left|\sinh \left(V_{s}\right)\right| d s \leq y_{0} \int_{0}^{\infty} e^{\left|V_{s}\right|-2 s} d s .
$$

Coupling $\left(V_{s}\right)$ with $\left(\widetilde{B}_{s}\right)$ using Lemma A.1 and then using (A.5), we find that $\mathbb{P}_{z_{0}}^{\kappa, \rho}$-a.s., $\sup _{s \geq 0}\left(\left|V_{s}\right|-s\right)<\infty$. Thus, $\mathbb{P}_{z_{0}}^{\kappa, \rho}$-a.s., $\int_{0}^{T_{z_{0}}} \frac{\left|X_{t}\right|}{X_{t}^{2}+Y_{t}^{2}} d t<\infty$, which implies that the limit $\lim _{t \rightarrow T_{z_{0}}^{-}} \int_{0}^{t} \frac{X_{r}}{X_{r}^{2}+Y_{r}^{2}} d r$ exists and is finite. Thus, $\mathbb{P}_{z_{0}}^{\kappa, \rho}$-a.s., $\lim _{t \rightarrow T_{z_{0}}^{-}} \lambda_{t} \in \mathbb{R}$. 
Corollary A.1. Almost surely $\gamma\left(\left[0, T_{z_{0}}\right)\right)$ is bounded.

Proof. This follows from Proposition 3.1 (i) and Lemma 4.1 in [11.

Lemma A.2. For any bounded measurable $U \subset \overline{\mathbb{H}}, \mathbb{P}_{\kappa}$-a.s., $\Psi_{t}^{\kappa, \rho}(U) \rightarrow 0$ as $t \rightarrow \infty$.

Proof. Taking $e^{2 b}=t /\left(2\left|z_{0}\right|^{2}\right)$ in (A.6) for some $t>0$, we get

$$
\mathbb{P}_{z_{0}}^{\kappa, \rho}\left[T_{z_{0}}>t\right] \leq 2^{1+1 / \kappa}\left(t /\left|z_{0}\right|^{2}\right)^{-1 / \kappa}, \quad t>0 .
$$

Using (3.8), we get

$$
\mathbb{E}_{\kappa}\left[M_{t}^{\kappa, \rho}\left(z_{0}\right)\right]=G^{\kappa, \rho}\left(z_{0}\right) \mathbb{P}_{z_{0}}^{\kappa, \rho}\left[T_{z_{0}}>t\right] \leq 2^{1+\frac{1}{\kappa}}\left|z_{0}\right|^{\frac{\rho+2}{\kappa}+\frac{\rho^{2}}{8 \kappa}} \cdot t^{-\frac{1}{\kappa}}, \quad t>0 .
$$

Suppose $|z| \leq R$ for every $z \in U$. Then

$$
\mathbb{E}_{\kappa}\left[\Psi_{t}^{\kappa, \rho}(U)\right]=\int_{U} \mathbb{E}_{\kappa}\left[M_{t}^{\kappa, \rho}(z)\right] d A(z) \leq \pi 2^{\frac{1}{\kappa}} R^{2+\frac{\rho+2}{\kappa}+\frac{\rho^{2}}{8 \kappa}} \cdot t^{-\frac{1}{\kappa}}, \quad t>0 .
$$

Thus, $\lim _{t \rightarrow \infty} \mathbb{E}_{\kappa}\left[\Psi_{t}^{\kappa, \rho}(U)\right]=0$. Since $\left(\Psi_{t}^{\kappa, \rho}(U)\right)$ is a positive supermartingale, from Doob's martingale convergence theorem, we see that $\mathbb{P}_{\kappa}$-a.s., $\lim _{t \rightarrow \infty} \Psi_{t}^{\kappa, \rho}(U)$ exists. From Fatou's lemma, $\mathbb{E}_{\kappa}\left[\lim _{t \rightarrow \infty} \Psi_{t}^{\kappa, \rho}(U)\right] \leq \lim _{t \rightarrow \infty} \mathbb{E}_{\kappa}\left[\Psi_{t}^{\kappa, \rho}(U)\right]=0$. So we get the conclusion.

Remark. Proposition 3.1 (i) and Lemma A.2 also hold if $\frac{\kappa}{2}-4<\rho<\frac{\kappa}{2}-2$. In that case we may use the estimate $\left|V_{s}\right| \leq\left|V_{0}\right|+\sqrt{\kappa}\left|\widehat{B}_{s}\right|+\left(\rho+4-\frac{\kappa}{2}\right) s$, which follows from (A.3) and that $|\tanh (x)| \leq 1$ for $x \in \mathbb{R}$. Then we may apply (A.5) with $a \in\left(0, \frac{\kappa}{2}-2-\rho\right)$.

\section{References}

[1] L. V. Ahlfors. Conformal invariants: topics in geometric function theory. McGraw-Hill Book Co., New York, 1973.

[2] T. Alberts, S. Sheffield. The Covariant Measure of SLE on the Boundary, Probab. Theory Rel., 149:331-371, 2011.

[3] T. Alberts, S. Sheffield. Hausdorff dimension of the SLE curve intersected with the real line, Electron. J. Probab., 40:1166-1188, 2008.

[4] V. Beffara. The dimension of the SLE curves. Ann. Probab., 36(4):1421-1452, 2008.

[5] C. Benes, F. J. Viklund, G. Lawler. Conformal invariance of the loop-erased random walk intensity. arXiv:1402.7345, 2014.

[6] F. Camia, C. Newman. Critical Percolation Exploration Path and SLE(6): a Proof of Convergence. Probab. Theory Rel., 139:473-520, 2007.

[7] B. Duplantier, J.P. Miller and S. Sheffield. Liouville quantum gravity as a mating of trees. arXiv:1409.7055, 2014.

[8] L. Field. Two-sided radial SLE and length-biased chordal SLE. arXiv:1601.03374, 2016.

[9] L. Field and G. Lawler. Escape probability and transience for SLE. Electron. J. Probab., 20(10):1-14, 2015. 
[10] P. Jones and S. Smirnov. Removability theorems for Sobolev functions and quasiconformal maps. Ark. Mat., 38(2):263-279, 2000.

[11] G. Lawler. Conformally Invariant Processes in the Plane, Amer. Math. Soc., 2005.

[12] G. Lawler. Continuity of radial and two-sided radial SLE at the terminal point. In the Tradition of Ahlfors-Bers. VI. Contemp. Math., 590:101-124. Amer. Math. Soc., Providence, RI, 2013.

[13] G. Lawler. Minkowski content of the intersection of a Schramm-Loewner evolution (SLE) curve with the real line. J. Math. Soc. Japan, 67:1631-1669, 2015.

[14] G. Lawler and M. Rezaei. Minkowski content and natural parameterization for the Schramm-Loewner evolution. Ann. Probab., 43:1082-1120, 2015.

[15] G. Lawler and S. Sheffield. A natural parametrization for the Schramm-Loewner evolution. Ann. Probab., 39,:1896-1937, 2011.

[16] G. Lawler. O. Schramm, and W. Werner. Conformal invariance of planar loop-erased random walks and uniform spanning trees, Ann. Probab., 32:939-995, 2004.

[17] G. Lawler and F. J. Viklund. Convergence of loop-erased random walk in the natural parametrization. arXiv:1603.05203, 2016.

[18] G. Lawler and F. J. Viklund. The Loewner difference equation and convergence of loop-erased random walk. arXiv:1611.01406, 2016.

[19] G. Lawler and F. J. Viklund. Convergence of radial loop-erased random walk in the natural parametrization. arXiv:1703.03729, 2017.

[20] G. Lawler and W. Zhou. SLE curves and natural parametrization. Ann. Probab., 41:1556-1584, 2013.

[21] J. Miller and S. Sheffield. Imaginary Geometry III: reversibility of $\operatorname{SLE}_{\kappa}$ for $\kappa \in(4,8)$. Annals of Math., 184(2):455-486, 2016.

[22] J. Miller and S. Sheffield. Imaginary Geometry IV: interior rays, whole-plane reversibility, and space-filling trees. arXiv:1302.4738.

[23] D. Revuz and M. Yor. Continuous Martingales and Brownian Motion. Springer, Berlin, 1991.

[24] S. Rohde and O. Schramm. Basic properties of SLE, Ann. Math., 161:879-920, 2005.

[25] S. Rohde and D. Zhan. Backward SLE and the symmetry of the welding. Available at arXiv:1307.2532. To appear in Probab. Theory Rel.

[26] O. Schramm. Scaling limits of loop-erased random walks and uniform spanning trees. Israel J. Math., 118:221-288, 2000.

[27] O. Schramm and D. B. Wilson. SLE coordinate changes. New York Journal of Mathematics, 11:659-669, 2005.

[28] S. Smirnov. Critical percolation in the plane: Conformal invariance, Cardy's formula, scaling limits. C. R. Acad. Sci. Paris Ser. I Math. 333:239-244, 2001.

[29] D. Zhan. Reversibility of chordal SLE. Ann. Probab., 36(4):1472-1494, 2008.

[30] D. Zhan. Duality of chordal SLE. Invent. Math., 174(2):309-353, 2008.

[31] D. Zhan. Duality of chordal SLE, II. Ann. I. H. Poincare-Pr., 46(3):740-759, 2010.

[32] D. Zhan. Ergodicity of the tip of an SLE curve. Prob. Theory Related Fields, 164(1):333-360, 2016.

[33] D. Zhan. SLE loop measures. arXiv:1702.08026. 